\title{
Submicron Matrices Embedded in a Polymeric Caplet for Extended Intravaginal Delivery of Zidovudine ${ }^{i}$
}

\author{
Felix Mashingaidze ${ }^{1}$, Yahya E. Choonara ${ }^{1}$, Pradeep Kumar ${ }^{1}$, Lisa C. du Toit ${ }^{1}$, \\ Vinesh Maharaj ${ }^{2}$, Eckhart Buchmann ${ }^{3}$, Viness Pillay ${ }^{1 *}$ \\ ${ }^{1}$ Wits Advanced Drug Delivery Platform Research Unit, Department of Pharmacy and Pharmacology, \\ School of Therapeutic Sciences, Faculty of Health Sciences, University of the Witwatersrand, \\ Johannesburg, 7 York Road, Parktown, 2193, South Africa \\ ${ }^{2}$ Department of Chemistry, Faculty of Natural and Agricultural Sciences, University of Pretoria, \\ Pretoria 0002, South Africa \\ ${ }^{3}$ Department of Obstetrics and Gynaecology, Faculty of Health Sciences, University of the \\ Witwatersrand, Johannesburg, 7 York Road, Parktown, 2193, South Africa
}

\author{
*Corresponding Author: \\ Professor Viness Pillay \\ Tel: $+27-11-717-2274$ \\ Fax: $+27-11-642-4355$ \\ Fax2Email: +27-86-553-4733 \\ Email: viness.pillay@wits.ac.za
}

Running Title: Composite caplet for intravaginal zidovudine delivery

\section{Abstract}

In this study, an intravaginal delivery system able to deliver an anti-HIV-1 agent for the purpose of potentially reducing HIV-1 transmission acting over an extended duration was successfully formulated. This delivery system was a composite polymeric caplet comprising zidovudine-loaded polyethylene glycol enclatherated pectin-mucin submicron matrices embedded within a poly (D,L-lactide), magnesium stearate, Kollidon ${ }^{\circledR} \mathrm{SR}$ and Carbopol ${ }^{\circledR}$ 974P NF-based polymeric caplet matrix. A three-factor and three-level Box-Behnken statistical design was utilized to optimize the polymeric caplet. The optimized directlycompressed composite polymeric caplet hardness was $22.1 \pm 0.3 \mathrm{~N}$ and the matrix resilience was $62.4 \pm 0.6 \%$. The swelling- and diffusion-controlled fractional zidovudine (AZT) release from the optimized caplet was $0.74 \pm 0.01$ in simulated vaginal fluid (SVF), which increased to $0.81 \pm 0.21$ in phosphate-buffered saline (PBS) simulating seminal fluid, over 30 days. Caplet matrix swelling was directly related to the percentage Carbopol 974P NF composition. An intravaginal system for AZT delivery was tested in the pig model over 28 days. X-ray analysis depicted delivery system swelling with matrix contrast fading over time as vaginal fluid permeated the matrix core. Plasma, vaginal fluid swab eluates and tissue AZT

\footnotetext{
'This study was derived from the Master's dissertation of first author, Felix Mashingaidze, submitted 24 March 2014
} 
concentrations were measured by gradient ultra-performance liquid chromatography (UPLC)-tandem photodiode array detection. Vaginal tissue and vaginal fluid swab eluate AZT concentrations remained above effective levels over 28 days and were higher than plasma AZT concentrations, availing a system with reduced systemic toxicity and more effective inhibition of viral replication at the site of entry.

Keywords: zidovudine (AZT), intravaginal drug delivery, X-ray imaging, ultra-performance liquid chromatography, histopathology

\section{Introduction}

Development of topically-applied chemical agents that may effectively prevent vaginal HIV-1 transmission (microbicides) has been a major challenge for scientists (1-3). The difficulties have included microbicide acceptance as an alternative HIV-1 prevention modality, pharmacokinetic inadequacy whereby the microbicide has to be distributed throughout the vaginal mucosa in sufficient quantities to prevent HIV-1 transmission and mucosal toxicity which diminishes vaginal mucosa's barrier properties resulting in easy HIV-1 mucosal transcytosis and transmission $(2,3)$. Despite all these challenges recent notable advances in the field include demonstration of the safety, tolerability and pharmacokinetic clinical trial successes of various gel, film, and vaginal tablet formulations, and most significantly the dapivirine-loaded vaginal ring which is used over a month as demonstrated by clinical trials conducted by the International Partnership for Microbicides (IPA) and the Microbicide Trial Network (MTN) $(4,5)$. In particular, a Phase III ASPIRE trial of the dapivirine loaded ring that ran from 2012-2015 demonstrated a reduction in HIV-1 infection by $27 \%$ overall $(4,5)$. There is thus scope for further research on intravaginal devices to improve on the efficacy of already existing technologies and to explore novel drug delivery design concepts.

Motivation for further research is still present in that HIV/AIDS continues to be pandemic, new infections are still occurring each day, and use of microbicides may be advantageous in that they may be used discreetly by the female partner thus shifting the control of HIV-1 transmission prevention from the male to the female (6). Microbicides provide a frontline defense approach where HIV-1 is targeted at the vagina mucosal surface when it is still vulnerable since the quantity of virus that initially transcytoses the vaginal mucosa (founder population) has been shown to be inadequate to sustain transmission until viral replication occurs (3,6-9). Therefore, microbicides may act at this juncture to contain HIV-1 replication hence prevent transmission. Acting at the mucosal surface where fewer CD4 ${ }^{+}$cells are 
present as compared to the submucosa, microbicides have the capacity to curb HIV-1 transmission (8).

Microbicide potential capacities comprise the potential for incorporation of anti-HIV-1 agents with in vitro potency, microbicide success stories exemplified by the $39 \%$ success rate of the $1 \%$ tenofovir gel observed in the CAPRISA study, and the scientific knowledge accumulated thus far including the postulated reasons of the failure of some microbicides such as mucosal integrity disruption $(3,10)$. Microbicide research is now focused intensely on microbicide developmental aspects such as bioavailability enhancement (targeted and extended release formulations) and toxicological exclusion $(3,11,12)$.

Future microbicides would therefore be formulated to ensure that an appropriate level of the potent anti-HIV-1 agent is available at the vaginal mucosal interface and distributed throughout the entire target site for an extended duration of time to outlast HIV-1 (3). Strict toxicology and tolerance protocols using appropriate animal models would have to be followed to exclude irritancy and disruption of the vaginal mucosa barrier properties caused by the use of microbicides (13-16). Microbicide formulations have to satisfy these pharmacokinetic and pathotoxicity preconditions to achieve in vivo microbicide success $(2,3,6)$.

In this study a composite polymeric drug delivery system intended for intravaginal anti-HIV-1 agent delivery was tested in vivo over 28 days in the pig model. Zidovudine (AZT), the model agent, was encapsulated in polyethylene glycol enclatherated pectin-mucin (PEG-enclPEC:MUC) submicron matrices (SMMs), as previously investigated (17). AZT was used as a model drug, being a nucleotide reverse transcriptase inhibitor (NRTI). NTRIs have shown potential as ARV-based microbicide agents (18). NRTIs' potential application in microbicides was first demonstrated in a nonhuman primate model vaginal challenge (18). The CAPRISA 004 trial was the first efficacy trial of an ARV-containing microbicide demonstrating protection of women from HIV infection $(10,18)$. The SMMs formulated were then embedded within a polymeric caplet shaped matrix which was statistically optimized and evaluated in vitro, and thereafter inserted into the vaginal cavity of Large White female pigs. In vivo performance of the delivery system was observed by capturing X-ray images of the pig's pelvic region and $A Z T$ concentrations in vaginal fluid swab eluates, vaginal tissue and plasma were measured at predetermined time points over a period of 28 days. In addition, histopathological analysis was performed on pig vaginal tissue excised from the euthanized pigs after 28 experimental days. This study aimed at testing an alternative anti-HIV-1 agent delivery system in vivo, with the possibility of providing extended site-specific AZT delivery, 
with determination of the level of vaginal epithelia pathotoxicity that such a delivery system may cause. Incorporation of AZT within the mucoadhesive SMMs is proposed to promote the site-specific delivery to attain enhanced vaginal fluid and tissue concentrations versus plasma levels, and the caplet $\mathrm{pH}$-responsive mechanism could potentially enhance AZT release in the presence of alkaline semen, to potentially build on the successes of developed intravaginal devices such as the dapirivine ring. This delivery system also endeavors to address poor patient adherence challenges and enable potential non-disclosure of use to sexual partners since it is to be inserted discreetly once a month high into the posterior vaginal fornix where it will cause minimum interference during sexual intercourse, and will reside in the vaginal cavity delivering the anti-HIV-1 agent in a sustained and extended manner over an intended period of a month for prevention of HIV-transmission. In addition, use of this intravaginal caplet is less messy and is more aesthetically acceptable than other formulations such as gels. The concept of inserting an intravaginal tablet for disease treatment is an accepted practice, with products on the market for the treatment fungal infections and hormonal therapies in tablet form. This allays the potential of low acceptance of the delivery system by women $(4,5)$.

\section{Materials and Methods}

\subsection{Materials}

Poly $\mathrm{D}, \mathrm{L}$ lactide, Kollidon ${ }^{\circledR} \mathrm{SR}$, magnesium stearate, porcine gastric mucin, polyethylene glycol (PEG) 400, barium sulphate, acetonitrile and formic acid were obtained from Aldrich ${ }^{\circledR}$ (Sigma-Aldrich Inc., St. Louis, USA). Carbopol 974P NF was purchased from Noveon Inc, Cleveland, OH, USA. Pectin, GENU ${ }^{\circledR}$ pectin type USP/100 [Degree of esterification (DE) 5565\%] was obtained from CP Kelco ApS, Lille Skensved, Denmark. Active pharmaceutical ingredients (APIs); 1-[(2R,4S,5S)-4-azido-5-(hydroxymethyl)oxolan-2-yl]-5-methylpyrimidine2,4-dione commonly known as zidovudine (AZT) and 2',3'-dideoxy-3'-thiacytidine, commonly known as lamivudine (3TC) were obtained from GlaxoSmithKline, Middlesex, UK. UPLC grade water was purified using a Milli- $Q^{\circledR}$ gradient water purification system (Millipore SAS, Molsheim, France). Other materials and excipients employed were of analytical grade and were utilized as obtained.

\subsection{Preparation of the AZT-loaded composite polymeric caplet}

A composite polymeric delivery system composed of AZT-loaded submicron matrices (submicrospheres) embedded within a caplet-shaped polymeric matrix was prepared $(19,20)$. The AZT-encapsulating submicrospheres (SMMs) were formulated through a crosslinking-emulsion technique where a $\mathrm{Ca}^{2+}(0.01 \mathrm{M}, 3.89 \mathrm{~mL})$ crosslinked dispersion of 
pectin (270mg), porcine gastric mucin (30mg), polyethylene glycol $400(0.1 \mathrm{~mL})$, and AZT (300mg) acted as the aqueous phase, and cyclohexane was the oil phase. Optimized independent parameters of ultrasonication time $6.28 \mathrm{~min}$, drug:polymer ratio $1: 1$ and surfactant (Span® 85) concentration $1.64(\% / v)$ were utilized (17). The caplet shaped polymeric matrix was directly compressed from uniformly blended poly $D, L$ lactide (937.50mg), Kollidon ${ }^{\circledR}$ SR (75.00mg), magnesium stearate $(7.50 \mathrm{mg})$ Carbopol $^{\circledR}$ 974P NF $(2 \% \mathrm{w} / \mathrm{w})$ and the AZT-loaded SMMs $(20 \% \mathrm{w} / \mathrm{w})$. Measured quantities of poly (D,L-lactide (PDLL), magnesium stearate (MS), Kollidon ${ }^{\circledR}$ SR (KSR), AZT-loaded PEG-encl-PEC:MUC SMMs $(20 \% \mathrm{w} / \mathrm{w})$ and Carbopol 974P NF $(1 \% \mathrm{w} / \mathrm{w})$ were blended for 5 minutes into a uniform dry powder mixture. The powder was then directly compressed into a caplet in a custommade laboratory scale punch and die mould on a hydraulic Carver press (Carver Inc, Wabash, IN, USA) at a compression force of 2.5 tons. Different quantities of each ingredient were added in accordance with the formulations generated from the Box-Behnken statistical design.

\subsection{Box-Behnken design optimization of the composite polymeric caplet}

The three-factor, three-level $\left(3^{3}\right)$ Box-Behnken statistical design employed to optimize the fabrication of the composite polymeric caplet had upper and lower levels of three independent parameters; mass of PDLL, mass of MS and the mass of KSR as shown in Table I (21). The responses; fractional mass increase (FMI) after 7 days of caplet dissolution in simulated vaginal fluid (SVF), caplet matrix hardness $(\mathrm{H})$ and fractional drug released (FDR) after 7 days of caplet dissolution in SVF were determined (Table I). The 15 formulations generated by the Box-Behnken design (Table I) were experimentally tested and the results obtained were fed into the MINITAB $^{\circledR}$ software $\left(\right.$ MINITAB $^{\circledR}$, V15, Minitab, USA) which then computed the optimized formulation's independent parameters and expected responses. 
Table I. Box-Behnken design independent parameter limits, response objectives, and design template of the 15 polymeric caplet formulations

\begin{tabular}{|c|c|c|c|}
\hline & Variable & & its (mg) \\
\hline Independent par & & Lower & Upper \\
\hline Mass of poly $(D, L$ & tide) (mg) & 937.5 & 1102.5 \\
\hline Mass of magnesi & tearate $(\mathrm{mg})$ & 7.5 & 22.5 \\
\hline Mass of Kollidon & (mg) & 75.0 & 225.0 \\
\hline Response & & & jective \\
\hline Fraction of drug $\mathrm{r}$ & se at $t_{7 \text { days }}$ & & inimize \\
\hline Hardness (N) & & & aximize \\
\hline Fractional SMV* & $k e$ after $t_{7 \text { days }}$ & & inimize \\
\hline Formulation & $\begin{array}{l}\text { Mass of Poly (D,L- } \\
\text { lactide) (mg) }\end{array}$ & $\begin{array}{l}\text { Mass of magnesium } \\
\text { stearate }(\mathrm{mg})\end{array}$ & $\begin{array}{c}\text { Mass of Kollidon }^{\circledR} \\
\text { SR }(\mathrm{mg})\end{array}$ \\
\hline F1 & 1102.5 & 15.0 & 225.0 \\
\hline $\mathrm{F} 2$ & 1102.5 & 7.5 & 150.0 \\
\hline F3 & 1020.0 & 22.5 & 225.0 \\
\hline F4 & 937.5 & 15.0 & 225.0 \\
\hline F5 & 1020.0 & 7.5 & 225.0 \\
\hline F6 & 1102.5 & 22.5 & 75.0 \\
\hline F7 & 1020.0 & 22.5 & 75.0 \\
\hline F8 & 937.5 & 22.5 & 150.0 \\
\hline F9 & 1020.0 & 7.5 & 225.0 \\
\hline F10 & 937.5 & 7.5 & 150.0 \\
\hline F11 & 1020.0 & 15.0 & 150.0 \\
\hline F12 & 1102.5 & 15.0 & 75.0 \\
\hline F13 & 1020.0 & 15.0 & 150.0 \\
\hline F14 & 937.5 & 15.0 & 75.0 \\
\hline F15 & 1020.0 & 7.5 & 75.0 \\
\hline
\end{tabular}

\subsection{Drug release studies on the polymeric caplet}

The selection of a method for in vitro dissolution studies was a challenge given that dissolution of solid vaginal dosage forms has not been widely investigated. Some researchers recommend the use of dissolution testing methods used for suppositories (22). However, in this study, the generally preferred method of Gupta and co-workers (22) for in vitro assay for evaluation of solid vaginal dosage forms employing $100 \mathrm{~mL}$ dissolution media was adopted. Thus, drug release studies were performed for the AZT-containing composite polymeric caplet. The caplet was placed in a container filled with $100 \mathrm{~mL}$ of the SVF $(\mathrm{pH} 4.5)$ dissolution media (23-25). The container was placed in an orbital shaker incubator (Orbital Shaker Incubator, LM-530D, YIHDER TECHNOLOGY CO., LTD, Jhonghe City, Taipei County, Taiwan, Republic of China) which was kept at $37^{\circ} \mathrm{C}$ and rotating at $20 \mathrm{rpm}$. An aliquot $(200 \mu \mathrm{L})$ was withdrawn at predetermined time intervals of $3,7,14,21$ and 28 days and an equal volume of fresh SVF was replaced after each withdrawal. The fraction of drug released from the caplet was then computed from the UV absorbance values measured on $4 \mu \mathrm{L}$ pipette samples of the withdrawn aliquots at $25^{\circ} \mathrm{C}$ using a nanophotometer which was 
set to measure UV absorbance at $\lambda_{\max }$ of $267 \mathrm{~nm}$. Drug release studies were performed in triplicate. Drug release studies were also performed in phosphate buffered saline (PBS pH 7.4) which acted as a simulant for semen (26). The mean dissolution time (MDT) computed from the drug release data using Eq. 1 revealed the extent to which AZT release from the composite polymeric caplet could be extended. The larger the value of the MDT the longer drug release period was extended and vice versa.

$M D T=\frac{\sum_{i=1}^{n} t_{i} M_{t}}{M_{\infty}}$

Where; MDT is the mean dissolution time, $M_{t}$ is the amount of drug in time $t$ and $M_{\infty}$ is the loading dose.

A comparison was made of the dissolution efficiency (DE), calculated according to Eq. 2, of AZT in two different media, SVF ( $\mathrm{pH} 4.5)$ and PBS ( $\mathrm{pH} 7.4)$.

$$
D E=\frac{\int_{0}^{t} y d t}{y_{100}} \times 100 \%
$$

Where; $\mathrm{DE}$ is the dissolution efficiency and $\mathrm{y}$ is the percentage of drug dissolved in time $\mathrm{t}$.

The difference factor $\left(f_{1}\right)$ and the similarity factor $\left(f_{2}\right)$, calculated according to Eqs. 3 and 4 respectively, were used to compare the drug release profiles of the delivery system in two different dissolution media, SVF and PBS $(27,28)$. The difference factor $\left(\mathrm{f}_{1}\right)$ values close to $0 \%(0-15 \%)$ and similarity factor $\left(f_{2}\right)$ values above $50 \%(50-100 \%)$ indicate that the dissolution profiles being compared are similar.

$$
f_{1}=\frac{\sum_{t=1}^{n}\left|R_{t}-T_{t}\right|}{\sum_{t=1}^{n} R_{t}} \times 100 \%
$$

Where; $f_{1}$ is the difference factor, $R_{t}$ is the reference assay at time $t, T_{t}$ is the test assay at time $t$ and $n$ is the number of sampling points.

$$
f_{2}=50 \log \left\{\left[1+1 / n \sum_{t=1}^{n} W_{t}\left(R_{t}-T_{t}\right)^{2}\right]^{-0.5} \times 100\right\}
$$


Where; $f_{2}$ is the similarity factor, $W_{t}$ is the optional weight factor, $R_{t}$ is the reference assay at time $t, T_{t}$ is the test assay at time $t$ and $n$ is the number of sampling points.

Regression analysis was performed in an effort to determine the fit of the dissolution profile of the drug delivery system in SVF and PBS with known kinetic models (24). The coefficient of determination ( $\mathrm{R}^{2}$ adjusted) obtained from fitting the dissolution profile of the delivery system into 14 known kinetic models gave an indication as to which drug release kinetic model dissolution from the delivery system followed (24).

\subsection{Polymeric caplet matrix texture profiling}

Caplet matrix hardness was tested using a calibrated texture analyzer (Texture Analyzer TA.TX plus, Stable Microsystems, Surrey, UK) fitted with a $5 \mathrm{~kg}$ load cell. The compression test mode was employed, with a force target of $4 \mathrm{~N}$ and trigger of $0.05 \mathrm{~N}$. The peak compression force was measured in triplicate on three different locations along the caplet length using a $2 \mathrm{~mm}$ flat cylindrical probe and a mean peak compression force value was computed and regarded as a measure of the matrix hardness of the caplet. Measurements were performed on all the caplets fabricated according to the 15 Box-Behnken design template formulations (Table I) and on the optimized caplet. In addition, the percentage caplet matrix resilience was also determined (29).

\subsection{Effect of the quantity of Carbopol 974P NF on caplet matrix dimensions during dissolution}

The effect of the amount (percentage of the total caplet mass) of Carbopol 974P NF on the dimensional increase of the polymeric caplet after exposure to SVF for a month were measured at $1 \%, 2 \%$ and $3 \%$ Carbopol 974P NF content in the caplet. Caplets were immersed for 30 days in $100 \mathrm{~mL}$ SVF filled containers which were placed in an orbital shaker incubator (Orbital Shaker Incubator, LM-530D, YIHDER TECHNOLOGY CO., LTD, Jhonghe City, Taipei County, Taiwan, Republic of China) which was kept at $37^{\circ} \mathrm{C}$ and rotated at 20rpm. The effect of a silk pouch employed for in vivo investigations in the pig model (as discussed in Section 2.7) on caplet dimensional changes was also investigated for the 3\% Carbopol 974P caplet (greatest swelling potential) under the same conditions, with inclusion of the caplet in the pouch. The caplet dimensional increase of the caplet was then computed using Eq. 5.

$\%$ dimensional increase $=\frac{\left(D_{2}-D_{1}\right)}{D_{1}} \times 100$ 
where $D_{1}$ is the length, width or thickness of the caplets before being immersed in SVF for 30 days and $D_{2}$ is the length, width or thickness of the caplet after it was immersed in SVF for 30 days.

\subsection{In vivo experimental design and procedure in the Large White Pig model}

In vivo experimentation involved intravaginal insertion of a composite polymeric drug delivery system in 10 Large White female pigs weighing approximately $45 \mathrm{~kg}$. This in vivo animal study was approved by the Animal Ethics Screening Committee (AESC) of the University of the Witwatersrand, Johannesburg, South Africa (AESC clearance No. 2011/44/05). The 10 pigs were divided into two groups of $5(n=5)$, with one group having the AZT-loaded delivery system (group B) inserted intravaginally whilst the other group (group A, control) had a placebo (drug-free) delivery system inserted. It became evident during this study that there are anatomical and physiological differences between the pig vaginal cavity and the human vaginal cavity. In the pig, the cervix is continuous with the vaginal cavity and the urethra opens into the vaginal cavity whilst in the human vagina, the urethra and vagina run parallel with two different orifices and the cervix protrudes into the vaginal cavity forming fornices of which the posterior vaginal fornix was the proposed area where the delivery system was to be placed. Gynecological observations also highlighted the increased moisture of the pig vaginal environment leading to accelerated expulsion. The use of the netted silk pouch, sutured to the vaginal wall, in this study was to ensure that the delivery system was retained in the vaginal cavity for the entire 28 day duration of the study (which would not be a requirement for human placement). The maximum retention time in the pig vaginal cavity without the use of the netted silk pouch was only two hours as it was expelled from the vagina by the pigs, hence the need to use the netted silk pouch sutured to the vaginal wall. The pouch was netted to allow free movement of vaginal fluid into and out of the pouch thus minimizing its effect on drug release from the delivery system. Silk material was chosen for the pouch in this study in an effort to minimize toxicity based of the biocompatibility profile of silk-based biomaterials (32). Studies were undertaken on dimensional changes in the caplet (Section 2.6) to demonstrate that the netted silk pouches used in this study were large enough to accommodate the delivery system even after maximum swelling after a month in SVF or PBS.

Each pig was anesthetized by an intramuscular injection of ketamine $(11 \mathrm{mg} / \mathrm{kg}$ ) and midazolam $(0.3 \mathrm{mg} / \mathrm{kg})$ on the thigh muscle. The pig was then laid in the ventral position on an operating table where it was maintained under general anesthesia using inhalation $2 \%$ isoflurane and $12 \%$ medical oxygen $(30,31)$. The composite polymeric delivery system was enclosed in a netted silk pouch that was then secured in the vaginal cavity by suturing the 
netted silk pouch onto the vaginal mucosa at a position after the urethra and proximal to the cervix.

Throughout experimentation the pig's cardiac activity (electrocardiogram), pulse, blood pressure and temperature were monitored (30). In addition, the pig's weight was measured and recorded every week.

\subsubsection{In vivo $X$-ray imaging of the intravaginal polymeric delivery system}

The composite polymeric delivery system was formulated to contain uniformly dispersed barium sulphate $(10 \% \mathrm{w} / \mathrm{w})$, a radio opaque material which enabled X-ray detection of the delivery system in situ. The pig was laid on an operating table in the dorsal position whilst under anesthesia and an X-ray of its pelvic region was taken using a Shimadzu MobileArt Evolution X-ray system (model MUX 200, Shimadzu Corporation, Kyoto, Japan). X-ray images of the delivery system were taken on days; 3, 7, 14, 21 and 28 after the delivery system was inserted in the vaginal cavity in order to detect its presence and location intravaginally, as well as to observe any dimensional changes (31).

\subsubsection{Vaginal fluid swab eluate collection}

Vaginal swabbing was performed to collect vaginal fluids whilst the pig was under anesthesia before the insertion of the delivery system and on days 3, 7, 14, 21 and 28 after delivery system insertion employing cotton buds for sample collection. A consistent procedure of swabbing was followed: one cotton bud was inserted $4 \mathrm{~cm}$ into the vaginal cavity for 1 minute and then swabbing of the vaginal wall in one outward stroke was performed on removing the cotton bud from the vagina with care taken not to contact the delivery system. Cotton buds were weighed before and after swabbing in order to account for weight variability (33). Each sample cotton bud was placed in $2 \mathrm{~mL}$ Eppendorf tubes and then stored at $-80^{\circ} \mathrm{C}$ until analysis. This swabbing technique was employed in an effort to ensure reproducibility and validity of the experiment.

\subsubsection{Blood sampling}

Blood samples $(10 \mathrm{~mL})$ were withdrawn directly from the internal jugular vein whilst the anesthetized pig laid in the dorsal position starting on the day of delivery system insertion (day 0 , before delivery system insertion) followed by blood sampling on days 3, 7, 14, 21 and 28 after the delivery system was inserted in the vaginal cavity $(31,33)$. Withdrawn blood samples were immediately placed in heparinized tubes which were centrifuged at 1200rpm and the supernatant was stored in $2 \mathrm{~mL}$ Eppendorf tubes at $-80^{\circ} \mathrm{C}$ for further analysis. 


\subsubsection{Vaginal tissue sample collection}

On day 28 , after X-ray imaging, vaginal swab collection and blood sampling, the pigs were euthanized by means of an intravenous injection of Phenobarbital $(200 \mathrm{mg} / \mathrm{kg})$ to one of the ear veins to enable vaginal tissue collection. The vaginal tract was excised from the pelvic canal after midline dissection through the symphysis pubis. Thereafter the vaginal tract was dissected to expose the vaginal mucosa. Two tissue samples were then collected from each pig, one for quantitative bioanalysis to determine AZT tissue concentration and the other for histopathology analysis. Tissue samples for quantitative tissue AZT determination were placed in tubes and stored at $-80^{\circ} \mathrm{C}$ until analysis whist the tissues for histopathology assessment were fixed in $10 \%$ neutral-buffered formalin till analysis $(14,15)$.

\subsection{Quantitative chromatographic determination of AZT in plasma, vaginal fluid and vaginal tissue}

\subsubsection{Instrumentation and chromatographic conditions}

Detection and quantification of AZT in porcine plasma samples was performed on a Waters Acquity ${ }^{\mathrm{TM}}$ ultra performance liquid chromatographic (UPLC) system (Waters Corporation, Milford, Massachusetts, USA) consisting of a binary solvent manager, a sample manager and fitted with a photodiode array detector (PDA) synchronously controlled using the Waters Empower 2 software which collected and processed the chromatography data (34). An Acquity UPLC ${ }^{\circledR}$ HSS T3 1.8 $\mu \mathrm{m} ; 2.1 \times 150 \mathrm{~mm}$ analytical column (Waters Corporation, Dublin, Ireland), fitted subsequent to a VanGuard ${ }^{\mathrm{TM}}$ Pre-column 3/Pk 2.1 $\times 5 \mathrm{~mm}$ column (Waters Corporation, Dublin, Ireland), was used to detect and separate AZT from the internal standard (IS), 3TC. Chromatographic separation was performed in the gradient mode at a flow rate of $0.5 \mathrm{~mL} / \mathrm{min}$ with the eluent composed of varying proportions of $0.2 \%$ formic acid in water $(A)$ and acetonitrile $(B)$.

Chromatographic separation was performed at a $40^{\circ} \mathrm{C}$ column and sample temperature and UV detection was set at $267 \mathrm{~nm}$, the AZT $\lambda_{\max }$. Samples were filtered through $0.2 \mu \mathrm{m}$ membrane filters (GHP Acrodisc ${ }^{\circledR} 13 \mathrm{~mm}$ Syringe Filters, Pall Corporation, New York, USA) and placed into $1.5 \mathrm{~mL}$ Waters glass vials kept at room temperature in the sample compartment. Sample volume used during analysis was $2 \mu \mathrm{L}$ and total analysis runtime was 3 minutes.

\subsubsection{Preparation of diluent, stock and working standard solutions}

Acetonitrile:water $(50: 50 \mathrm{v} / \mathrm{v})$ was prepared and used as the diluent. AZT stock solution $(100 \mu \mathrm{g} / \mathrm{mL})$ was prepared by dissolving $10 \mathrm{mg}$ of AZT into $100 \mathrm{~mL}$ of the diluent in a 
volumetric flask and the working standard solutions were prepared by serial dilutions of the stock solution to obtain solutions of concentrations $10-100 \mu \mathrm{g} / \mathrm{mL}(\mathrm{n}=10)$.

\subsubsection{Solid phase extraction and measurement of AZT from plasma}

Plasma samples $(500 \mu \mathrm{L})$ placed in $2 \mathrm{~mL}$ Eppendorf tubes were pre-treated to remove proteins by the addition of $1 \mathrm{~mL}$ acetonitrile (35). The mixture was vortexed for 2 minutes then centrifuged for a further 2 minutes at 1200rpm [Table Top High Speed Centrifuge (TG16-WS), Hunan Xiangyi Laboratory Instrument Development Co., Ltd, Hunan, China]. The supernatant was transferred to new $2 \mathrm{~mL}$ Eppendorf tubes and zinc sulphate $(500 \mu \mathrm{L}$, $0.1 \% \mathrm{w} / \mathrm{v}$ ) was added and then vortexed and centrifuged again and the supernatant transferred to new $2 \mathrm{~mL}$ Eppendorf tubes. Thereafter, the organic phase was evaporated at $30^{\circ} \mathrm{C}$ under a nitrogen stream to obtain samples to be used in the solid phase extraction (SPE) procedure (36).

SPE was performed under vacuum using Waters Oasis ${ }^{\circledR}$ HLB $3 \mathrm{cc}$ (60mg) extraction cartridges mounted on a 20 hole Waters glass block vacuum manifold processing station (Waters Corporation, Milford, Massachusetts, USA). The Oasis ${ }^{\circledR}$ HLB cartridges were preconditioned by first passing methanol $(2 \mathrm{~mL})$ through followed by Milli-Q water $(2 \mathrm{~mL})$ at $5 p s i$ then the pre-treated plasma samples were passed through the column with minimum suction to allow maximum drug adsorption onto the Oasis ${ }^{\circledR}$ HLB cartridge. Finally the drug was eluted using different solvents. The solvent with the highest elution efficiency (high drug recovery) among methanol, ethanol and acetonitrile was the elution solvent of choice and used throughout the study. The eluates were dried at $30^{\circ} \mathrm{C}$ under a nitrogen stream and the residue was reconstituted with the diluent $(1 \mathrm{~mL})$, filtered through $0.2 \mu \mathrm{m}$ membrane filters (GHP Acrodisc ${ }^{\circledR} 13 \mathrm{~mm}$ Syringe Filters, Pall Corporation, New York, USA) and placed into the $1.5 \mathrm{~mL}$ Waters glass vials for UPLC-PDA analysis.

\subsubsection{Analysis of AZT concentration in plasma, vaginal fluid swab eluates and tissue samples of group B pigs}

Frozen plasma samples obtained on days $0,3,7,14,21$ and 28 were allowed to thaw over $24 \mathrm{hrs}$ and subjected to the SPE as described in Section 2.8.3 then UPLC-PDA AZT detection and quantification was performed.

After defrosting vaginal swab samples (cotton buds) for $24 \mathrm{hrs}$, $1 \mathrm{~mL}$ diluent (acetonitrile:water 50:50) was added to the Eppendorf tubes containing the vaginal swab samples to extract AZT. The samples were vortexed for $5 \mathrm{~min}$ then centrifuged at $1200 \mathrm{rpm}$ and the supernatant was subjected to SPE as described before AZT detection and 
quantification using UPLC-PDA, taking into account cotton swab sample weights. To measure the amount of free AZT in the excised vaginal tissue for each pig in group $B$, frozen samples ( $1 \mathrm{~g}, 3$ samples from each pig, $\mathrm{n}=3$ ) were separately ground in a coffee grinder into a fine tissue sample, placed in $2 \mathrm{~mL}$ Eppendorf tubes then diluent $(1 \mathrm{~mL}$, acetonitrile:water 50:50) was added as the AZT extraction solvent (33). The samples were vortexed for 10min, centrifuged at $1200 \mathrm{rmp}$ and the collected supernatant was subjected to SPE (Section 2.8.3) before AZT detection and quantification by UPLC-PDA.

\subsection{Histopathology analysis of porcine cervicovaginal epithelia}

Histopathology tests were performed to determine the level of abnormality (such as inflammation and toxicity) that may have been caused by the presence of a AZT-releasing polymeric delivery system in the vaginal cavity over 28 days $(33,37)$. Excised vaginal tissue from groups A (5 pigs, polymeric delivery system with no drug) and B (5 pigs, drug-loaded polymeric delivery system) were fixed in $10 \%$ normal buffered formalin sections $(5-6 \mu \mathrm{m})$ were cut and stained with Haematoxylin and Eosin (H/E) for microscopy analysis (13-15,33).

The type of vaginal epithelia, thickness of the epithelial cell layer, degree of inflammation, ulceration, fibrosis and mononuclear inflammatory infiltration as well as tissue necrosis, fibrosis and integrity of the lamina propria and submucosa were examined. Different areas (8-10 fields) of the vaginal mucosa were examined and scored blindly for histopathological changes such as epithelial ulceration, epithelial proliferation and epithelial exocytosis (inflammatory cell influx into the epithelium), subepithelial leukocytic influx, stromal fibrosis, perivascular cell cuffing and tissue necrosis. The assigned histopathology scores were: 0 , no abnormality; 1, minimum abnormality, 2, mild abnormality; 3, moderate abnormality; 4, severe abnormality (15). A comparison was then undertaken of the histological observations for the vaginal tissue excised from group $A$ and $B$ pigs to vaginal tissue excised from a control group of 5 large white pigs (Group C) used in an earlier similar study conducted in our laboratories by Ndesendo and co-workers (38) where no delivery system was inserted into the vagina of the pigs throughout the 28 day study (38).

\section{Results}

\subsection{Fabrication and optimization of the composite polymeric caplet}

The polymeric caplets fabricated by direct compression according to the 15 Box-Behnken design formulations had variable masses and dimensions depending on the amount of material that was used to fabricate them. The optimized formulation caplet had an approximate length of $25 \mathrm{~mm}$, width of $10 \mathrm{~mm}$ and thickness of $6 \mathrm{~mm}$. The experimental 
design yielded varying results of FMI, $\mathrm{H}$ and FDR (Table II). These results were then inputted into the MINITAB $^{\circledR}$ software to yield an optimized formulation with a composite desirability (D) of 0.997 , fractional mass increase desirability $\left(d_{F M I}\right)$ of 1.000 , hardness desirability $\left(d_{H}\right)$ of 1.000 and fractional drug release desirability $\left(d_{F D R}\right)$ of 0.991 . The optimum independent parameters were computed to be; PDLL mass of $937.5 \mathrm{mg}$, MS mass of $7.5 \mathrm{mg}$ and KSR mass of $75.0 \mathrm{mg}$. These independent parameters yielded computed responses of: FMI of $0.279, \mathrm{H}$ of 21.8 , and FDR of 0.401 . The relationships between the independent parameters and responses assessed by linear regression using MINITAB $^{\circledR}$ were described by the polynomial Eqs. 6,7 and $8(21)$, where the coefficient of determination of $\mathrm{FMI}\left(\mathrm{R}_{\mathrm{FMI}}^{2}\right)$ was $87.3 \%, \mathrm{H}\left(\mathrm{R}_{\mathrm{H}}^{2}\right)$ was $61.7 \%$ and that of FDR $\left(\mathrm{R}_{\mathrm{FDR}}^{2}\right)$ was $77.9 \%$.

$F M I=1.616-0.002[P D L L]+0.668[M S]-0.001[K S R]$

$H=-14.465+0.101[P D L L]-0.706[M S]-0.109[K S R]-0.005\left[M S^{*} M S\right]+0.001\left[P D L L^{*} M S\right]+0.001\left[M S^{*} K S R\right]$

$F D R=3.964-0.008[P D L L]+0.009[M S]+0.007[K S R]$

Table II. Experimental responses to the 15 design formulations used in the optimization of the polymeric caplet

\begin{tabular}{cccc}
\hline Formulation & $\begin{array}{c}\text { Fractional mass } \\
\text { increase after 7days in } \\
\text { SVF }^{a}\end{array}$ & Hardness (N) & $\begin{array}{c}\text { Fractional drug release } \\
\text { in SVF after } \\
\text { 7 days }\end{array}$ \\
\hline F1 & 0.320 & 20.2 & 0.227 \\
F2 & 0.314 & 18.4 & 0.245 \\
F3 & 0.678 & 21.6 & 0.346 \\
F4 & 0.502 & 18.4 & 0.376 \\
F5 & 0.474 & 19.8 & 0.369 \\
F6 & 0.358 & 19.6 & 0.332 \\
F7 & 0.394 & 20.0 & 0.274 \\
F8 & 0.635 & 21.0 & 0.419 \\
F9 & 0.314 & 18.6 & 0.264 \\
F10 & 0.404 & 21.5 & 0.320 \\
F11 & 0.386 & 21.4 & 0.288 \\
F12 & 0.330 & 20.2 & 0.330 \\
F13 & 0.366 & 21.3 & 0.261 \\
F14 & 0.507 & 21.0 & 0.328 \\
F15 & 0.284 & 20.2 & 0.243 \\
\hline a-simulated vaginal fluid & & &
\end{tabular}

\subsection{Drug release from the polymeric caplet}

The fraction of AZT released from the polymeric caplet was measured over 7 days for the 15 design formulations and results obtained are presented in Table II. The fraction of drug released varied from 0.227 in formulation $\mathrm{F} 1$ to 0.419 in formulation F8 (Table II). The 
optimized formulation had a computed fractional drug release within 7 days of 0.401 . The fraction of drug released from the optimized formulation was then experimentally tested in both SVF and PBS (acted as a semen simulant) over 30 days to obtain the drug release profiles depicted in Fig. 1. The fraction of drug released after 30 days in SVF pH 4.5 was $0.743 \pm 0.010$ and that released in PBS pH 7.4 was $0.806 \pm 0.210$. The caplet thus significantly retarded release of $A Z T$ compared to fractional AZT release from the non-entrapped SMMs previously presented, being $\sim 0.91$ and $\sim 0.94$ in SVF and PBS after 24 hours, respectively (17).

The effect of the dissolution media $\mathrm{pH}$ in conjunction with the $1 \% \mathrm{w} / \mathrm{w}$ Carbopol 974P NF on the caplet dissolution kinetics as illustrated in Fig. 1 was confirmed by the similarity factor $\left(f_{2}=44.8\right)$ and the difference factor $\left(f_{1}=28.7\right)$ whose computed values show that the dissolution profile obtained in SVF is different from that obtained in PBS.

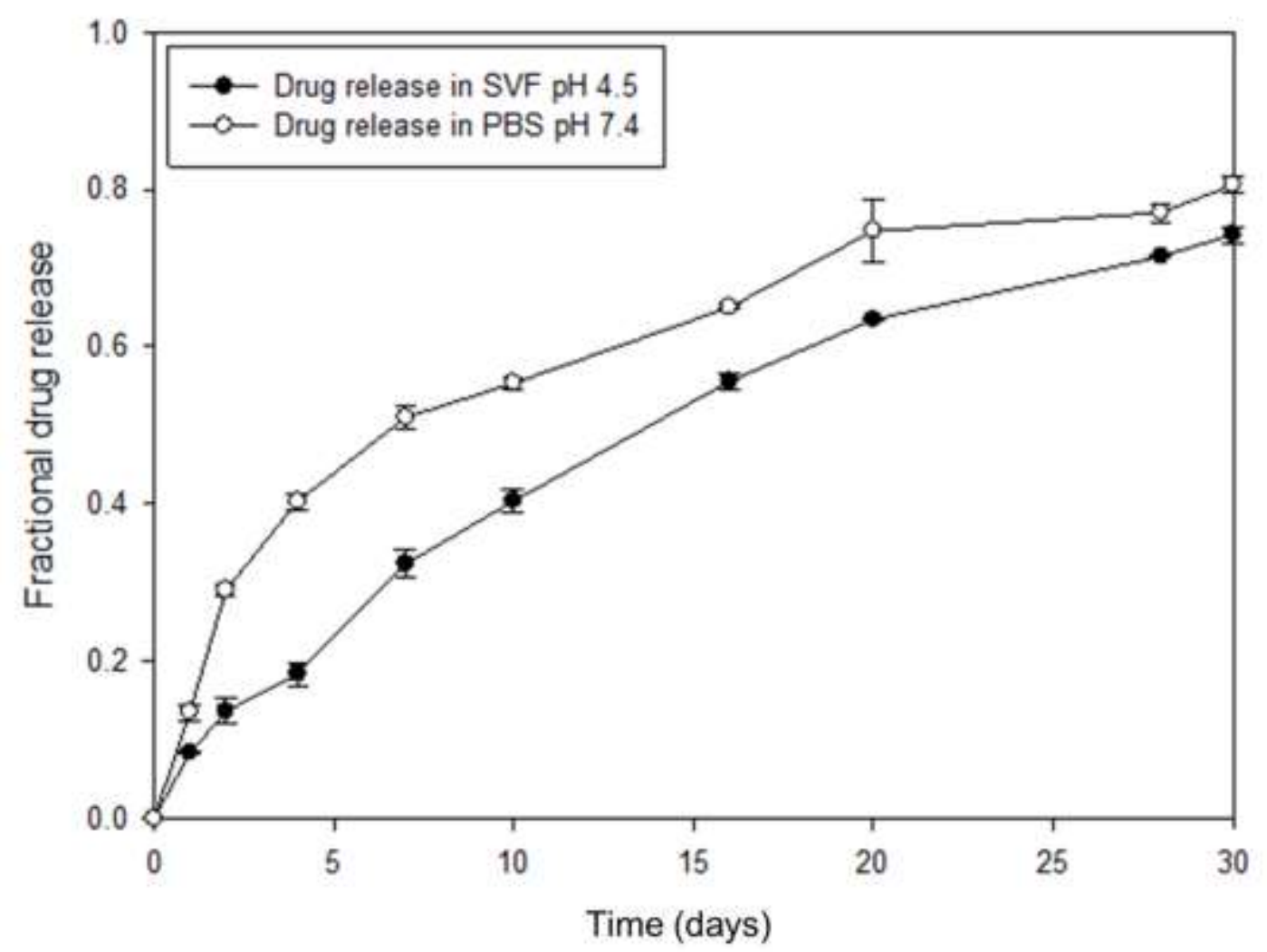

Fig. 1. Fractional AZT release from the optimized caplet formulation in SVF and PBS

The drug release mechanism, in both SVF and PBS, from the composite polymeric caplet was shown to follow most kinetic models it was fitted into as given by the adjusted coefficient

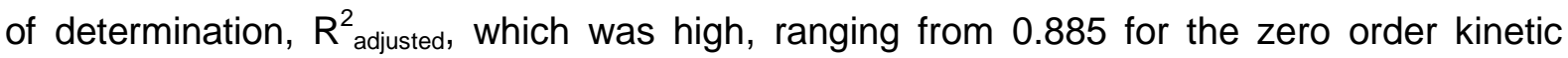


model to 0.997 for the Makoid-Banakar model in SVF and from 0.502 for the zero order kinetic model to 0.995 for the Weibull model in PBS (Table III).

\subsection{Effect of the quantity of Carbopol 974P NF on caplet matrix swelling}

The amount of Carbopol 974P NF in the caplet determined the degree of SVF or PBS uptake and hence the caplets' percentage dimensional increase (swelling, increase in length, width and thickness). Fig. 2a depicts digital photographs of the polymeric caplets containing $1 \%, 2 \%$ and $3 \% \mathrm{w} / \mathrm{w}$ Carbopol 974P NF before and after being immersed in SVF for a month. The percentage dimensional increase in length, width and thickness of the caplet are illustrated by the bar graph in Fig. $2 b$. There was no significant effect $(p<0.005)$ of the pouch on dimensional changes of the polymeric caplet containing the highest level of Carbopol 974P when placed in the silk pouch compared with the dimensional changes of the caplet not enclosed within the pouch.

Fig. 3 shows a digital photograph (a) and scanning electron micrographs (SEMs) (b-f) of cross-sectional area of the caplet before and after dissolution in SVF or PBS for 30 days, highlighting the increase in swelling and porosity with hydration and Carbopol 974P NF content.

\subsection{Polymeric caplet matrix textural analysis}

Caplet matrix hardness $(\mathrm{H})$ values for the 15 design formulations were measured and are presented in Table II. Caplet hardness varied from $18.4 \mathrm{~N}$ in formulation $\mathrm{F} 11$ to $21.6 \mathrm{~N}$ in formulation F15 (Table II). The optimized formulation had a Box-Behnken computed caplet matrix hardness of $21.8 \mathrm{~N}$ whilst the experimentally measured hardness was $22.1 \pm 0.3 \mathrm{~N}$.

In addition, the percentage matrix resilience of the caplet was computed from the force versus time curve according to Eq. 9 (29).

$$
\text { Matrix resilience }(\%)=\frac{\int_{2}^{3} f(t) d t}{\int_{1}^{2} f(t) d t} \times 100
$$

Where $\int_{2}^{3} f(t) d t$ is second (descending) area and $\int_{1}^{2} f(t) d t$ is the first (ascending) area under the curve. The mean percentage polymeric caplet matrix resilience was $62.4 \pm 0.6 \%$. 
Table III. Best fit parameters and adjusted coefficients of determination obtained after fitting the optimized caplet drug release profile to different kinetic models $(20,24)$

\begin{tabular}{|c|c|c|c|c|c|c|c|c|}
\hline \multirow{2}{*}{$\begin{array}{l}\text { Dissolution model } \\
\text { Zero order }\end{array}$} & \multicolumn{6}{|c|}{ Best-fit parameter } & \multicolumn{2}{|c|}{$\begin{array}{l}\text { Goodness of fit } \\
\text { parameter } \\
\left(R^{2} \text { adjusted }\right)\end{array}$} \\
\hline & $\begin{array}{l}\text { Parameter } 1 \\
\text { pH } 4.5 \\
\end{array}$ & $\begin{array}{l}p H 7.4 \\
k_{n}=3.29\end{array}$ & $\begin{array}{l}\text { Parameter } 2 \\
\text { pH } 4.5\end{array}$ & pH 7.4 & $\begin{array}{l}\text { Parameter } 3 \\
\text { pH } 4.5\end{array}$ & pH 7.4 & $\begin{array}{l}p H 4.5 \\
0.885\end{array}$ & $\begin{array}{l}p H 7.4 \\
0.502\end{array}$ \\
\hline First order & $k_{1} 0.050$ & $k_{1}=0.078$ & & & & & 0.990 & 0.883 \\
\hline Higuchi & $k H=13.3$ & $k H=16.0$ & & & & & 0.977 & 0.952 \\
\hline Korsmeyer-Peppas & $k K P=9.66$ & $k K P=22.1$ & $\mathrm{n}=0.609$ & $\mathrm{n}=0.388$ & & & 0.991 & 0.979 \\
\hline Hixson-Crowell & $k H C=0.14$ & $k H C=0.020$ & & & & & 0.973 & 0.803 \\
\hline Hopfenberg & $k H B=0.00$ & $\mathrm{kHB}=0.00$ & $\mathrm{n}=3016$ & $\mathrm{n}=5886$ & & & 0.989 & 0.868 \\
\hline $\begin{array}{l}\text { Baker-Lonsdale } \\
\text { Makoid-Banakar }\end{array}$ & $\begin{array}{l}k B L=0.004 \\
k M B=6.99\end{array}$ & $\begin{array}{l}\mathrm{kBL}=0.007 \\
\mathrm{kMB}=18.7\end{array}$ & $\mathrm{n}=0.842$ & $\mathrm{n}=\mathbf{0 . 5 4 5}$ & $k=0.017$ & $k=0.014$ & $\begin{array}{l}0.952 \\
0.997\end{array}$ & $\begin{array}{l}0.987 \\
0.989\end{array}$ \\
\hline $\begin{array}{l}\text { Peppas-Sahlin } \\
\text { Quadratic }\end{array}$ & $\begin{array}{l}k_{1}=-24.0 \\
k_{1}=-0.001\end{array}$ & $\begin{array}{l}k_{1}=19.7 \\
k_{1}=-0.002\end{array}$ & $\begin{array}{l}k_{2}=30.8 \\
k_{2}=0.049\end{array}$ & $\begin{array}{l}k_{2}=-1.20 \\
k_{2}=0.072\end{array}$ & $m=0.205$ & $m=0.576$ & $\begin{array}{l}0.993 \\
0.993\end{array}$ & $\begin{array}{l}0.989 \\
0.882\end{array}$ \\
\hline Weibull & $\alpha=889$ & $\alpha=3.62$ & $\beta=0.566$ & $\beta=0.513$ & $\mathrm{Ti}=0.545$ & $\mathrm{Ti}=0.686$ & 0.992 & 0.995 \\
\hline Logistic & $\alpha=-0.297$ & $\alpha=-0.164$ & $\beta=2.67$ & $\beta=1.99$ & & & 0.994 & 0.992 \\
\hline Gompertz & $\alpha=4.46$ & $\alpha=2.03$ & $\beta=1.72$ & $\beta=1.38$ & & & 0.974 & 0.988 \\
\hline Probit & $\alpha=-1.78$ & $\alpha=-1.01$ & $\beta=1.61$ & $\beta=1.22$ & & & 0.990 & 0.993 \\
\hline
\end{tabular}



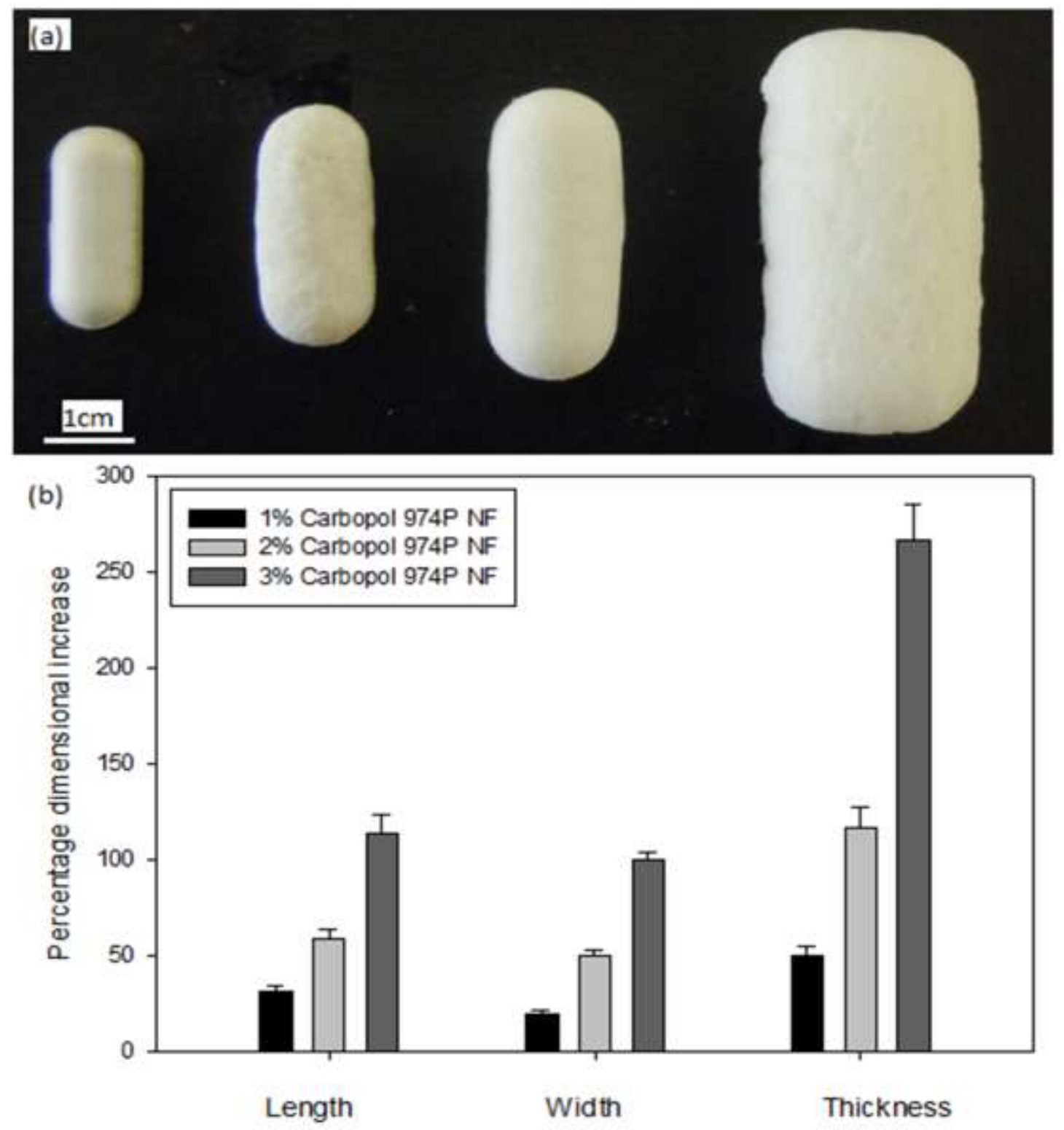

Fig. 2. (a) Digital photographs of polymeric caplets, from left to right; before exposure to SVF and after 30 days hydration in SVF for the caplets containing $1 \%, 2 \%$ or $3 \% \mathrm{w} / \mathrm{w}$ of Carbopol 974P NF (b) bar graph illustrating the different percentage dimensional increases in length, width and thickness of the polymeric caplet containing $1 \%, 2 \%$ and $3 \% \mathrm{w} / \mathrm{w}$ Carbopol 974P NF measured after the 30 day caplet dissolution in SVF 


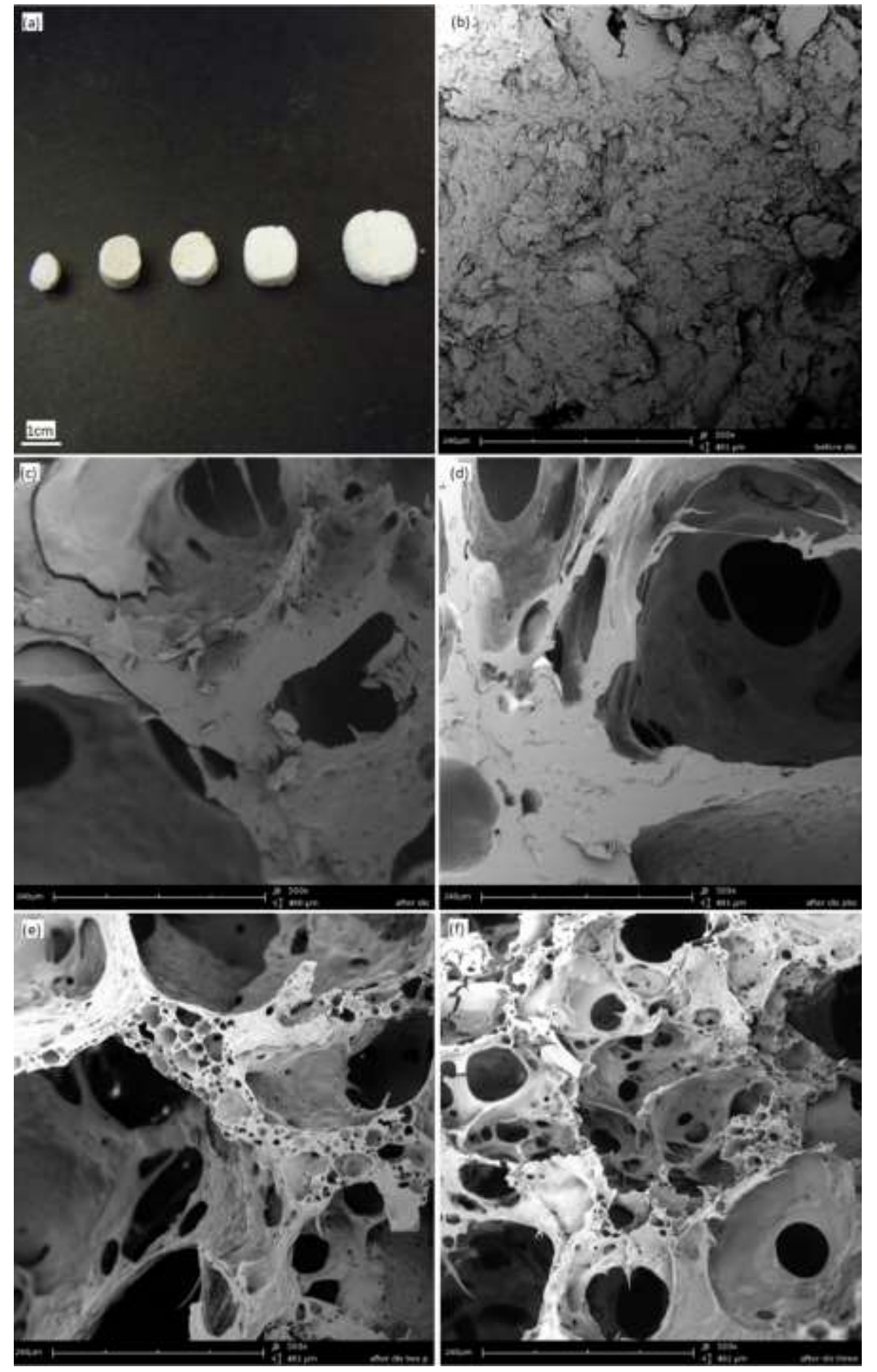

Fig. 3. (a) Digital photograph of the caplet's cross-section (from left to right); before dissolution and after $1 \% \mathrm{w} / \mathrm{w}$ Carbopol 974P NF containing caplet's dissolution in SVF and PBS, then $2 \%$ and $3 \% \mathrm{w} / \mathrm{w}$ Carbopol containing caplet's dissolution in SVF, respectively. (b) SEM micrograph acquired at 500X magnification of the caplet's cross-section before dissolution and (c-f) SEM micrograph of the caplet cross-section after a 30 day dissolution of the caplet containing, (c) $1 \% \mathrm{w} / \mathrm{w}$ Carbopol 974P NF in SVF (d) $1 \% \mathrm{w} / \mathrm{w}$ Carbopol 974P NF in PBS (e) $2 \% \mathrm{w} / \mathrm{w}$ Carbopol 974P NF in SVF and (f) $3 \% \mathrm{w} / \mathrm{w}$ Carbopol 974P NF in SVF 

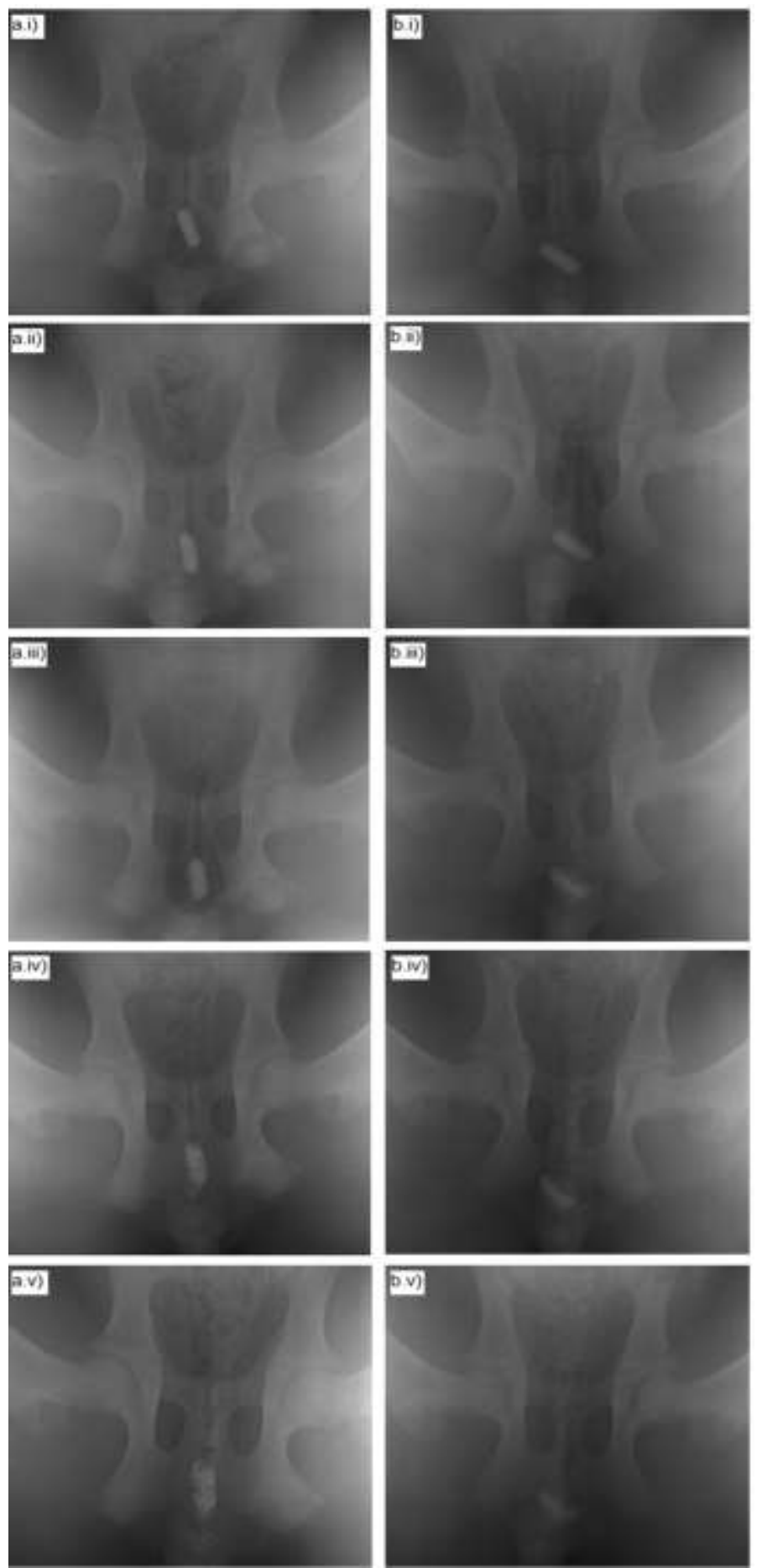

Fig. 4. X-ray images of the pig pelvic region depicting the composite polymeric delivery system with no drug observed on days, 3 (a.i), 7 (a.ii), 14 (a.iii), 21 (a.iv) and 28 (a.v), as well the AZT-loaded delivery system observed on days, 3 (b.i), 7 (b.ii), 14 (b.iii), 21 (b.iv) and 28 (b.v)

\subsection{In vivo X-ray imaging of the intravaginal polymeric delivery system}

The composite polymeric delivery system was observed in situ in the vaginal cavity by X-ray imaging of the pelvic region of the Large White pigs as depicted in Fig. 4. Figs. 4a.i-v (delivery system with no drug) show that the polymeric delivery system increasingly swells with increase in its residence time in the vaginal cavity. Figs. 4b.i-v depict the AZT loaded 
composite polymeric delivery system on days 3, 7, 14, 21 and 28 after it was inserted in the vaginal cavity. The delivery system in group B pigs was observed to increasingly fade in contrast, due to its erosion, from the outside inwards, with time.

\subsection{Quantitative chromatographic determination of AZT in porcine plasma, vaginal fluid swab eluates and vaginal tissue samples}

Analysis of chromatograms obtained for plasma, vaginal fluid and tissue samples showed suitable IS and analyte separation with the IS peak observed at retention time $\left(R_{t}\right) \approx 0.95 \mathrm{~min}$, AZT peak $R_{\mathrm{t}} \approx 1.85 \mathrm{~min}$. Additional smaller peaks that may be attributed to plasma, vaginal fluid and tissue constituents were also observed at $R_{t} \approx 0.8 \mathrm{~min}$ and $R_{t} \approx 1.5 \mathrm{~min}$.

AZT concentration in plasma and vaginal fluids computed from the area under the curve of analyte/IS $\left(A \cup C_{\text {analyte/IS }}\right)$ with reference to the calibration curve $\left(A \cup C_{\text {analyte/lS }}=0.608 t+0.977\right)$ for days 3, 7, 14, 21 and 28 were plotted against time and presented in Fig. 5. Generally, both plasma and vaginal fluid AZT concentrations were observed to decrease with time (Fig. $5 \mathrm{a}$ and b) with low AZT plasma concentrations ranging from $1.18 \pm 0.01 \mu \mathrm{g} / \mathrm{mL}$ to $1.13 \pm 0.02 \mu \mathrm{g} / \mathrm{mL}$. The highest plasma concentration $\left(C_{\max }\right)$ was obtained on day 7 (Fig. $\left.5 \mathrm{a}\right)$. AZT concentrations in vaginal fluid swab eluates (range: $5.00 \pm 0.98 \mu \mathrm{g} / \mathrm{mL}$ to $2.87 \pm 0.81 \mu \mathrm{g} / \mathrm{mL}$ ) were notably higher than those of plasma (Fig. $5 \mathrm{~b}$ ). Vaginal tissue AZT concentrations (range: $4.22 \pm 0.34 \mu \mathrm{g} / \mathrm{mL}$ to $2.96 \pm 0.19 \mu \mathrm{g} / \mathrm{mL}$ ) achieved after 28 days were higher than plasma AZT concentrations and were in the same range as vaginal fluid concentrations (Fig. 5c). 

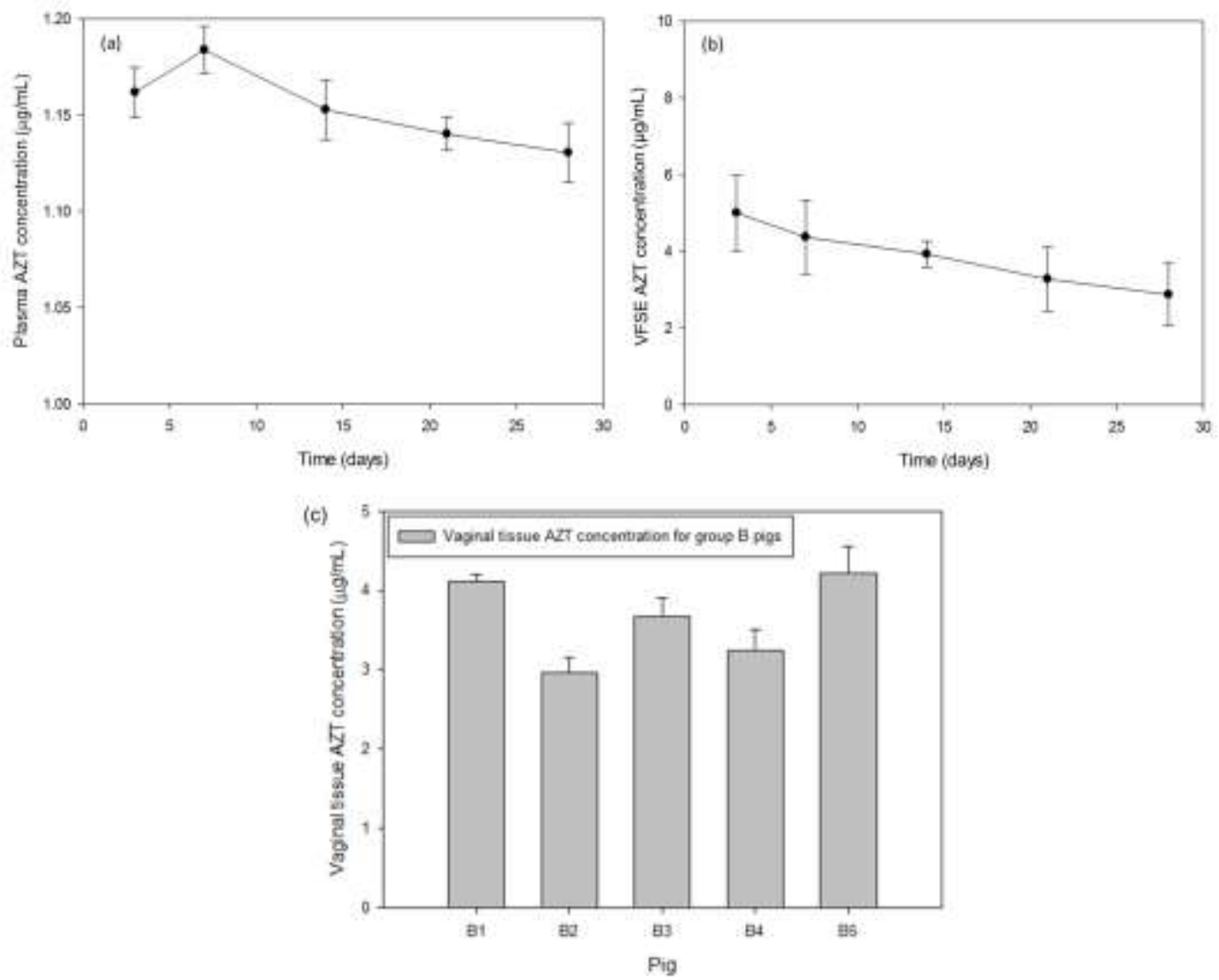

Fig. 5. AZT concentration measured in: (a) plasma and (b) vaginal fluid swab eluate (VFSE) samples collected on days; $3,7,14,21$ and 28 from group B pigs; and (c) vaginal tissue samples excised from group $B$ pigs after the 28 day study

\subsection{Histopathology analysis of porcine cervicovaginal epithelia}

Histological analysis of cut and formalin-fixed sections (Fig. 6) of the excised porcine vaginal mucosa after extended vaginal delivery of AZT by means of a composite polymeric intravaginal drug delivery system resulted in the scored abnormality observations that include epithelial erosion/ulceration, epithelial proliferation, epithelial exocytosis, subepithelial leukocyte influx, stromal fibrosis, perivascular cell cuffing and tissue necrosis as shown in Table IV and Fig. 6 where these observations are compared to the scored histological observations of excised normal porcine vaginal mucosa from a control group of 5 large white pigs (Group C) used in an earlier similar study in our laboratories by Ndesendo and co-workers (38), and presented in Table IV. 
Table IV. Histopathological observations of the vaginal sections excised from 5 pigs from group A and 5 pigs from group B (44) compared with 5 pigs from a control group (C) as reported by Ndesendo et al. (38)

\begin{tabular}{|c|c|c|c|c|c|c|c|c|c|c|c|c|c|c|c|}
\hline \multirow{2}{*}{$\begin{array}{l}\text { Group } \\
\text { Pig identity }\end{array}$} & \multicolumn{5}{|c|}{ A } & \multicolumn{5}{|c|}{$\mathrm{B}$} & \multicolumn{5}{|c|}{ C [Source: Ndesendo et al. (38)] } \\
\hline & $\mathrm{A} 1$ & $\mathrm{~A} 2$ & A3 & A4 & A5 & B1 & B2 & B3 & B4 & B5 & C1 & C2 & C3 & C4 & C5 \\
\hline Epithelial erosion/ulceration & - & $1+$ & $3+$ & $4+$ & - & - & - & - & $1+$ & $2+$ & - & - & - & - & - \\
\hline Epithelial proliferation & $2+$ & $1+$ & - & - & - & - & - & $1+$ & $2+$ & $2+$ & - & - & - & - & - \\
\hline Epithelial exocytosis & $3+$ & $4+$ & $4+$ & $4+$ & $1+$ & $3+$ & $3+$ & $1+$ & $3+$ & $4+$ & - & - & - & - & - \\
\hline Subepithelial leukocyte influx & $3+$ & $4+$ & $4+$ & $4+$ & $1+$ & $2+$ & $2+$ & $1+$ & $3+$ & $4+$ & $1+$ & & & & $1+$ \\
\hline Stromal fibrosis & - & - & $1+$ & $1+$ & - & - & - & - & $2+$ & $2+$ & - & - & - & - & - \\
\hline Perivascular cell cuffing & $1+$ & $2+$ & $3+$ & $3+$ & $1+$ & $1+$ & $1+$ & - & $3+$ & $4+$ & - & - & - & - & - \\
\hline $\begin{array}{l}\text { Tissue necrosis } \\
\text { - no abnormality }\end{array}$ & - & - & - & - & - & - & - & - & $3+$ & - & - & - & - & - & - \\
\hline $1+$ minimal abnormality & & & & & & & & & & & & & & & \\
\hline $2+$ mild abnormality & & & & & & & & & & & & & & & \\
\hline $3+$ moderate abnormality & & & & & & & & & & & & & & & \\
\hline $4+$ severe abnormality & & & & & & & & & & & & & & & \\
\hline
\end{tabular}



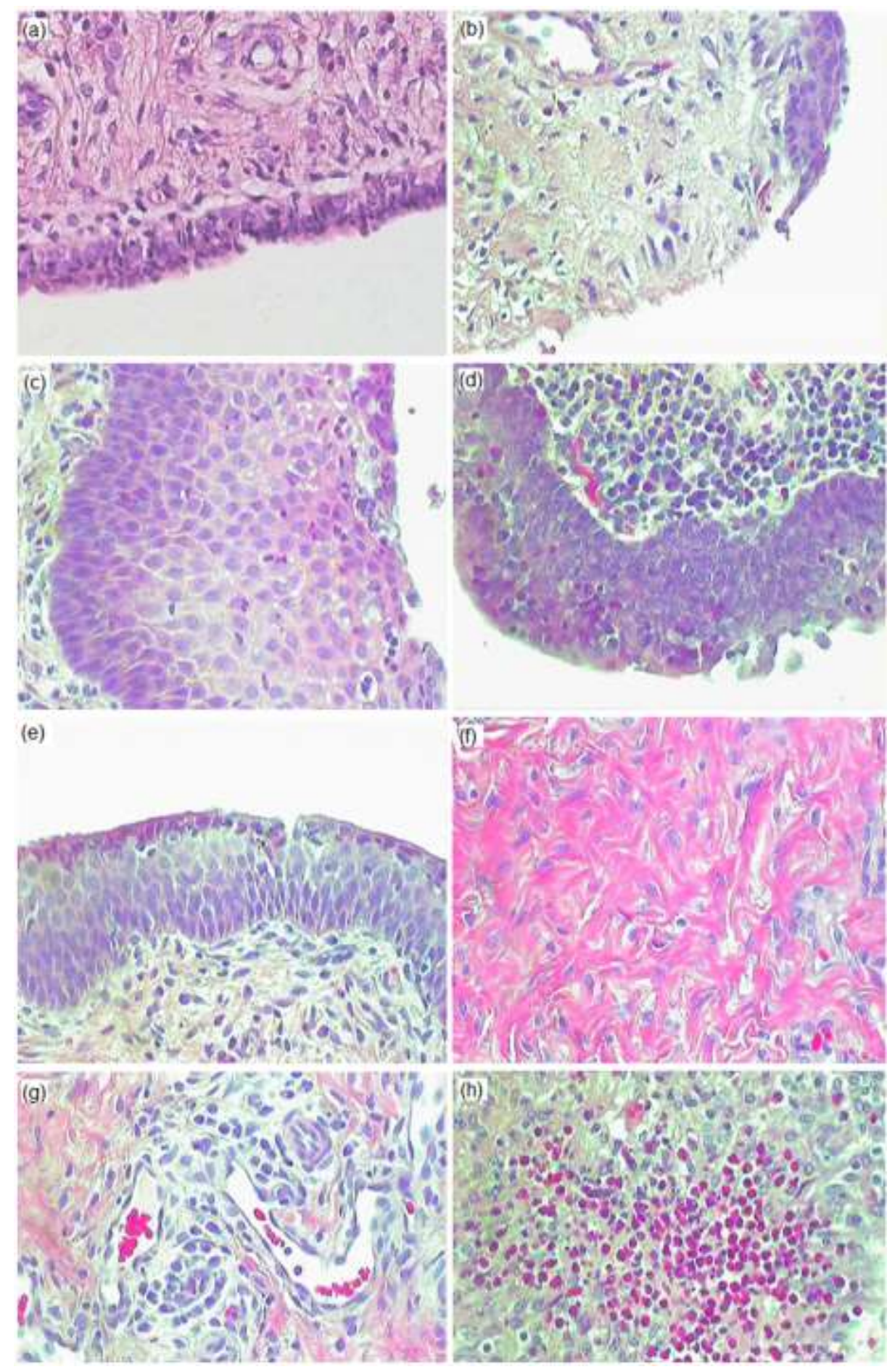

Fig. 6. Histopathology images of excised pig vaginal tissue depicting: (a) normal epithelia and lamina propria (b) epithelial erosion/ulceration, pig B4, (c) epithelial proliferation, pig A1, (d) epithelial exocytosis, pig A4, (e) subepithelial leukocyte influx, pig B4, (f) stromal fibrosis, pig B5, (g) perivascular cell cuffing, pig B4 and (h) tissue necrosis and polymorphonuclear exudation, pig B4

\section{Discussion}

\subsection{Drug release from the optimized polymeric caplet}

There was a MDT decrease when dissolution was performed in PBS (MDT=7.68 days) as compared to in SVF (MDT=10.6 days) corresponding to the marginal decrease in the 
fraction of drug released in SVF ( $\mathrm{pH} 4.5)$ to that released in PBS ( $\mathrm{pH} \mathrm{7.4)} \mathrm{(Fig.} \mathrm{1).} \mathrm{This} \mathrm{may}$ be attributed to the $1 \%$ Carbopol composition of the polymeric caplet. Carbopol 974P NF is $\mathrm{pH}$-responsive due to the several carboxylic groups it possesses which are ionized in basic media (PBS pH 7.4) and unionized in acidic media (SVF pH 4.5). Therefore, these ionized carboxylic groups, in basic media $(\mathrm{pH} 7.4)$, might have repelled each other and polarized as well as attracted the dissolution media's water molecules resulting in an increase in dissolution media uptake leading to caplet matrix swelling as depicted in Fig. 2. This matrix destabilization and increase in basic dissolution media uptake by the caplet then resulted in an increase in drug release and a decrease in MDT at an increased $\mathrm{pH}$ (39). This is favorable for further stimulating release of AZT from the caplet in the presence of seminal fluid, which is a high risk period for HIV transmission.

The effect of dissolution media $\mathrm{pH}$ on the release from the caplet was ascertained from the similarity and difference factors. Since $\mathrm{f}_{2}$ values from 50-100 ensure sameness of dissolution profiles and $f_{1}$ values from $0-15$ ensures little difference in the dissolution profiles, values of $f_{2}$ (44.8) and $f_{1}$ (28.7) were not within these ranges which indicates that the dissolution profiles were different (28). The increase in DE when dissolution was performed in PBS (DE=59.6\%) as compared to dissolution in SVF where DE was $48.1 \%$ also confirms that the caplet dissolution displays $\mathrm{pH}$ responsiveness $(24,27,28)$.

The AZT release mechanism from the caplet was a sum of the release mechanism from the macromolecular SMMs and that from the main polymeric matrix. Drug release from the matrix was extended due to the hydrophobic PDLL whose hydrolytic degradation is slow (40). Dissolution media was drawn into the interior of the caplet by the hydrogen bond forming Carbopol 974P NF and the polyvinylpyrolidine component of KSR. Polyvinylpyrolidine was then dissolved and leached out of the caplet matrix together with the drug released from the SMMs, leaving pores. The pores formed would enable more dissolution media to enter the caplet matrix leading to more percolation-based AZT release (41). Drug release from the caplet matrix was therefore swelling- and diffusion-dependent.

\subsection{Effect of the quantity of Carbopol 974P NF on caplet matrix swelling}

An increase in the content of Carbopol 974P NF correlated with an increase in the hydroxyl and carboxyl groups in the caplet which would lead to more SVF or PBS being drawn into the caplet through the possible formation of hydrogen bonds between the hydroxyl and carboxyl groups of Carbopol 974P NF and those of the water molecules in SVF or PBS resulting in caplet matrix swelling. The alkaline PBS $(\mathrm{pH} 7.4)$ led to the ionization and repulsion of carboxyl groups resulting in more swelling than in the acidic SVF $(\mathrm{pH} 4.5)$ where 
the carboxyl groups remained neutral as depicted in Figs. 2 and 3. Similar phenomena are expected in vivo where normal vaginal fluid is acidic and seminal fluid is alkaline $(23,26)$. In addition, increase in Carbopol 974P NF concentration would therefore affect the drug release rate (39).

Fig. 3a clearly illustrates the positive effect of Carbopol 974P NF on the swelling of the polymeric caplet, with caplets containing more Carbopol $974 \mathrm{NF}(2 \%$ and $3 \% \mathrm{w} / \mathrm{w})$ having larger cross-sectional areas. Scanning electron micrographs (SEMs) following dissolution (Figs. 3c-f) reveal that more pores formed within the caplet compared to the unhydrated caplet (Fig. 3b) with swelling of the caplet, and that porosity increased with Carbopol 974P NF content. The pores observed might have been due to the bulk erosion of the caplet matrix that possibly involved the hydrolytic degradation of poly ( $D, L$-lactide) and the solubilization and leaching out of the polyvinylpyrolidine component of Kollidon ${ }^{\circledR}$ SR $(40,41)$.

\subsection{Polymeric caplet matrix textural analysis}

From the hardness response values of the 15 design formulations in Table II, it was observed that hardness decreased with increasing percentage content of PDLL and generally increased with an increase in the percentage content of the plastically-deforming KSR. The percentage composition of MS in the caplet had an ambiguous effect on caplet hardness as previously reported by Matsuda and co-workers (42). Both relatively low and high amounts of MS resulted in decreased caplet hardness.

\subsection{In vivo X-ray imaging of the intravaginal polymeric delivery system}

Figs. 4a.i-v (delivery system with no drug) show that the polymeric delivery system increasingly swells with increase in its residence time in the vaginal cavity. The swelling was caused by the absorption of vaginal fluids by the Carbopol 974P NF and Kollidon ${ }^{\circledR}$ SR which constitute the polymeric delivery system. Figs. 4b.i-v depict the AZT loaded composite polymeric delivery system on days $3,7,14,21$ and 28 after it was inserted in the vaginal cavity. The delivery system in group B pigs was observed to increasingly fade in contrast, from the outside inwards, with time, which may have been due to drug and polymer solubilization and possibly the formation of pores within the polymeric delivery system as a result of the solubilization of the polyvinyl pyrolidone component of Kollidon ${ }^{\circledR} \mathrm{SR}$ as vaginal fluids penetrated the interior of the delivery system. The drug-loaded delivery system was observed to swell more than the delivery system with no drug. This was potentially due to the drug, AZT, which is mildly hydrophilic, which dissolved as vaginal fluid permeated into the polymeric delivery system matrix and percolated out. Due to caplet erosion, the caplet would not need to be removed. 


\subsection{Quantitative chromatographic determination of AZT in porcine plasma, vaginal fluid and vaginal tissue samples}

Chromatographic assay validation showed that acetonitrile was more efficient than methanol and ethanol at eluting the analyte, with an analyte recovery of $87.2 \%$. Mean recoveries $(n=3)$ calculated for analytes of concentrations: 1, 5 and $10 \mu \mathrm{g} / \mathrm{mL}$, increased with increasing analyte concentration (from 78.0 to $94.0 \%$ ). The computed limit of detection (LOD) and limit of quantification (LOQ) were $1.10 \mu \mathrm{g} / \mathrm{mL}$ and $3.35 \mu \mathrm{g} / \mathrm{mL}$, respectively. Intra-day and inter-day precision (RSD) ranged from $1.25 \%$ to $4.89 \%$ and $0.465 \%$ to $2.85 \%$ respectively whilst intraday and inter-day accuracy ( $R E$ ) ranged from $1.82 \%$ to $18.8 \%$, and $3.48 \%$ to $14.9 \%$, respectively. Both precision and accuracy were acceptable since they were less than $20 \%$ (34). Chromatographic conditions employed [column and sample temperature of $40^{\circ} \mathrm{C}$, light exposure, diluent of acetonitrile:water (50:50) and eluent, $0.2 \%$ formic acid water and acetonitrile] to detect and quantify AZT did not affect the stability of the analyte and the internal standard as their peak shapes remained similar and their retention times did not significantly shift when tested after $0,12,24,48$ and 72 hours.

AZT concentrations in the vaginal fluid swab eluates and tissue (Fig. $5 b$ and c) were higher than those of plasma (Fig. 5a). There is retention of AZT entrapped in the SMMs of the caplet in the vaginal cavity, which would still be required to permeate through the vaginal mucosa following diffusion from the SMMs in the caplet into the vaginal fluid in order for the drug to reach the blood (plasma). Thus, owing to the vaginal site-specific extended controlled release mechanism of the caplet, and the comparatively larger plasma volume, lower AZT plasma levels were achieved.

It must be noted that the main limitation to therapeutic effectiveness of AZT is its toxicity, specifically hematological toxicity (anemia and neutropenia), which are concentrationdependent. It has been demonstrated that hematopoietic progenitor cells (blood cell precursors) are inhibited by AZT at low concentrations. Thus plasma, vaginal fluid swab eluates and vaginal tissue AZT levels achieved in this study over the 28 day period of $1.18 \pm 0.01 \mu \mathrm{g} / \mathrm{mL}$ to $2.87 \pm 0.81 \mu \mathrm{g} / \mathrm{mL}, \quad 5.00 \pm 0.98 \mu \mathrm{g} / \mathrm{mL}$ to $2.87 \pm 0.98 \mu \mathrm{g} / \mathrm{mL}$, and $4.42 \pm 0.34 \mu \mathrm{g} / \mathrm{mL}$ to $2.96 \pm 0.19 \mu \mathrm{g} / \mathrm{mL}$, respectively, compare favorably with the mean $50 \%$ in vitro inhibitory concentration (ID50) of $0.44 \mu \mathrm{g} / \mathrm{mL}(0.24-0.64 \mu \mathrm{g} / \mathrm{mL}$ ), and the EC50 of $0.077 \pm 0.028 \mu \mathrm{M}(0.0206 \pm 0.0075 \mu \mathrm{g} / \mathrm{mL})$ and EC95 of $0.81 \pm 0.09 \mu \mathrm{M}(0.217 \pm 0.024 \mu \mathrm{g} / \mathrm{mL})$ $(43,44)$. The EC50 and EC95 represent the plasma concentration/AUC required for obtaining $50 \%$ and $95 \%$ of the maximum effect in vivo. Thus plasma levels achieved are effective yet low enough to limit toxicity, while localized vaginal fluid and tissue concentrations are higher and maintained for the duration of the study period (28 days), without a drop to 
subtherapeutic concentrations, to enable more effective inhibition of viral replication at the site of entry. More studies are, however, required to specify the actual concentrations required for efficacy in preventing HIV infection at the vaginal site.

The decreasing trend observed in vaginal AZT concentrations may have been due to the fact that vaginal fluid has to penetrate the polymeric matrix of the delivery system, dissolve AZT out of the SMMs and percolate out of the caplet matrix into the vaginal fluid. Therefore, with the time, vaginal fluid had to permeate through a longer distance into the matrix interior and percolate out with increasing resistance from the hydrophobic part of the polymeric matrix (poly $\mathrm{D}, \mathrm{L}$ lactide and the ethyl acetate component of the Kollidon ${ }^{\circledR} \mathrm{SR}$ ). A corresponding decreasing trend is also observed in plasma AZT concentration.

It is important to note that the $\mathrm{pH}$ in the pig vagina is approximately 7 unlike the human vagina which is approximately 4.5 (45). Therefore, in vivo intravaginal drug release from the delivery device in the pig model would be enhanced and will most likely follow the in vitro drug release profile simulated in PBS ( $\mathrm{pH} 7.4)$. In vivo AZT concentrations in human subjects are thus anticipated to be slightly lower under normal vaginal conditions, but enhanced on exposure to the higher $\mathrm{pH}$ of seminal fluid.

\subsection{Histopathology analysis of porcine cervicovaginal epithelia}

Histological observation of the excised vaginal tissue of pigs in the control Group $\mathrm{C}$ of Ndesendo and co-workers (38) (Table IV) in which no device was inserted generally showed no abnormalities except for minimum abnormality of subepithelial leukocyte influx observed in two of the five pigs (38). In some pigs (A5 and B1-B3), the vaginal epithelia and lamina propria were normal or had minimum abnormality (Table IV, Fig. 6a). On microscopy examination superficial loss of epithelial cells (epithelial erosion) was observed in 3 pigs from group A, and 2 pigs from group B (Table IV, Fig. 6b). The observed erosion of the porcine vaginal epithelia might have been caused by irritation, inflammatory process, leukocyte influx and exocytosis into the epithelia as a result of the presence of the drug delivery system. The epithelial erosion in pigs in group A may have been slightly enhanced as compared to that of pigs in group B because the delivery system in group A pigs was not drug-loaded hence did not swell to the same extent and did not allow vaginal fluid to infiltrate its interior as it did in the delivery system in the experimental group that had a hydrophilic drug encapsulated within them. This resulted in the delivery system in group A pigs remaining more solid and rigid due to the absence of the drug as compared to group $B$ caplets, which might have led to the slight increase in abrasiveness, hence increased group A pig epithelial erosion. 
Epithelial erosion is unfavorable because it diminishes the barrier properties of the vaginal epithelia and my lead to an increase in HIV-1 transcytosis and transmission.

Epithelial proliferation (due to epithelial hyperplasia) normally associated with low-grade subacute to chronic irritation was found to be mild to moderate in both groups (Table IV, Fig. $6 c$ ). The observed increased level of leukocyte influx (epithelial exocytosis) may have been a result of inflammation as was observed in the vaginal mucosa of most pigs (Table IV, Fig. $6 d)$. In the lamina propria directly underneath the vaginal epithelium, inflammatory infiltrates (subepithelial leukocyte influx), mainly neutrophils, some eosinophils and macrophages were detected in almost all vaginal mucosa examined with scoring varying from minimum to severe abnormality signifying minimum to severe inflammation (Table IV, Fig. 6e). There was more severe inflammation in group A pig vaginal epithelia as compared to group $B$ epithelia (Table IV). Inflammation of the vaginal mucosa weakens the epithelial barrier properties leading to possible HIV-1 transmission.

Stromal fibrosis, observed as fibroplasia in the deeper layers of the lamina propria underneath the vaginal epithelium was scored minimum to mild in the epithelial sections examined (Table IV, Fig. 6f). Perivascular cell cuffing identified as lymphocytic infiltrates and small nodules of lymphocytic perivascular inflammation was detected and scored as minimum to moderate in the epithelial sections examined except for one (B5) which was scored as severe (Table IV, Fig. 6g). On one vaginal epithelial section examined (B4), a focal area of muscle and tissue necrosis with glaucomatous inflammation was observed (Table IV, Fig. 6h). Within the area of tissue necrosis, numerous mononuclear cells as well as polymorphonuclear exudation were found and macrophages as well as few multinucleated giant cells could be identified. There seemed to be an infectious granuloma in the wall of the porcine vagina in pig B4. Tissue necrosis in pig B4 might have been an isolated case.

The use and suturing of the netted silk pouch might have adversely contributed to the histological observations noted, although this was not exclusively examined in this study.

\section{Conclusion}

This study successfully optimized (via a Box-Behnken design) and tested a composite polymeric delivery system in the pig as a model for extended vaginal AZT delivery intended to prevent HIV-1 transmission. The delivery system was observed in situ over 28 days and $\mathrm{X}$-ray showed that the drug loaded delivery system swelled and eroded gradually with time 
once vaginal fluid permeated into its matrix core. An UPLC-photodiode array assay was developed and optimized for selectivity, recovery, linearity, precision, accuracy and analyte stability and used to determine AZT concentrations in plasma and vaginal fluid swab eluate samples obtained at predetermined time points over 28 days and in vaginal tissue samples excised on day 28 after experimentation.

Vaginal fluid swab eluate and plasma AZT concentrations were shown to decrease gradually over the 28 days and the plasma AZT concentrations were lower than those of the vaginal fluid swab eluate concentrations. AZT concentrations in excised vaginal tissue samples were relatively constant and were in the same range as those of the vaginal fluid swab elautes, and these were maintained above the ID50, EC50 and EC95 of AZT for the duration of the study. Drug kinetics in this study was positive since much of the drug was found in the vaginal fluid swab eluate and vaginal tissue samples which receive the first exposure to HIV1 attributed to the mucoadhesive SMMs and site-specific mechanism, and comparatively less drug was detected in the plasma suggesting less systemic side effects would be caused by the drug. However, further studies are required to ascertain the actual concentration of AZT that should be in the vaginal fluid, vaginal tissue and plasma, respectively, for successful HIV-1 prevention.

Histopathological analysis exposed different scores of abnormalities on the excised vaginal tissue compared to pig tissues with no vaginal caplet exposure. Although the majority of abnormalities were scored as minimum to moderate epithelial proliferation and exocytosis, subepithelial leukocyte influx, perivascular cell cuffing, there were isolated cases of epithelial erosion, stromal fibrosis and tissue necrosis which raises concern regarding compromising the barrier properties of the vaginal mucosa which may lead to HIV-1 transmission. More research is necessary to further improve pharmacokinetics of the incorporated agent and reduce pathotoxicity to enable application as an alternative female-controlled pharmaceutical tool for reducing HIV-1 transmission. This will be ensued with pharmacokinetic and efficacy studies in nonhuman primates and pilot human biostudies.

In conclusion a caplet delivery system which was able to act as a reservoir for a model antiHIV-1 agent and capable of delivering AZT with site specificity over an extended duration of at least one month was successfully formulated from biocompatible materials and tested in vitro. These positive findings have also been noted with the dapivirine ring $(4,5)$. This delivery system, used discretely in vivo, would potentially ensure favorable levels of the antiHIV-1 agent in the vaginal cavity (with lower systemic availability) to counter HIV-1 in the seminal fluids interposed into the vaginal cavity during coitus, with no frequent dosing 
required prior to or after each coital act. In addition, due to the $\mathrm{pH}$-responsive capabilities of the caplet, this delivery system provides for increased anti-HIV-1 agent release when the $\mathrm{pH}$ of the vaginal cavity is increased as a result of the presence of the normally alkaline seminal fluid. The vaginal caplet is proposed to be an acceptable delivery system, comparing favorably to other vaginal systems such as gels and films and sustained mucosal delivery devices.

\section{Acknowledgements}

We acknowledge the Council for Scientific and Industrial Research, South Africa (CSIR SA), the South African National Research Foundation (NRF) and the Faculty of Health Sciences, University of the Witwatersrand, Johannesburg, South Africa, for their financial support.

\section{Conflict of interest statement}

The authors declare that there are no existing conflicting interests.

\section{References}

1. Hendrix CW, Cao YJ, Fuchs EJ. Topical microbicides to prevent HIV: clinical drug development challenges. Annu Rev Pharm Toxicol. 2009;49:349-75.

2. Adams JL, Kashuba DM. Formulation, pharmacokinetics and pharmacodynamics of topical microbicides. Best Pract Res Clin Obstet Gynaecol. 2012;26:451-62.

3. Pillay V, Mashingaidze F, Choonara YE, du Toit LC, Buchmann E, Maharaj V, et al. Qualitative and quantitative intravaginal targeting: key to anti-HIV-1 microbicide delivery from test tube to in vivo success. J Pharm Sci. 2012;101:1950-68.

4. Organization site: International Partnership for Microbicides: Clinical Trials. http://www.ipmglobal.org (2017). Accessed 13 Jun 2017.

5. Organization site: Microbicide Trials Network: Studies. http://www.mtnstopshiv.org (2017). Accessed 13 Jun 2017.

6. Shattock R, Solomon S. Microbicides-aids to safer sex. Lancet. 2004;363:1002-3.

7. Stone A. Microbicides: a new approach to prevent HIV and other sexually transmitted infections. Nat Rev Drug Discov. 2002;1:977-84.

8. Haase AT. Perils at mucosal front lines for HIV and SIV and their hosts. Nat Rev Immunol. 2005;5:783-92.

9. Haase AT. Targeting early infection to prevent HIV-1 mucosal transmission. Nature. 2010;464:217-23.

10. Abdool Karim Q, Abdool Karim SS, Frohlich JA, Grobler AC, Baxter C, Mansoor LE, et al. Effectiveness and safety of tenofovir gel, an antiretroviral microbicide, for the prevention of HIV infection in women. Science. 2010;329:1168-74.

11. Klasse PJ, Shattock R, Moore JP. Anteretroviral drug-based microbicide to prevent HIV1 sexual transmission. Annu Rev Med. 2008;59:455-71.

12. D'Cruz OJ, Dong Y, Uckun FM. Potent dual anti-HIV and spermicidal activities of novel oxovanadium(IV) complexes with thiourea non-nucleoside inhibitors of HIV-1 reverse transcriptaseBiochem Biophys Res Commun. 2003;302:253-64. 
13. D'cruz OJ, Erbeck D, Uckun FM. A study of the pig as a model for the vaginal irritancy of benzakonium chloride in comparison to the non-irritant microbicide $\mathrm{PHI}-443$ and the spemicide vanadocene dithiocarbamate. Toxicol Pathol. 2005;33:465-76

14. Cone RA, Hoen T, Wong X, Abusuwwa R, Anderson DJ, Moench T. Vaginal microbicides: detecting toxicities in vivo that paradoxically increase pathogen transmission. BMC Infect Dis. 2006;1:90. doi:10.1186/1471-2334-6-90.

15. D'cruz OJ, Uckun FM. Evaluation of local tolerance of the antiretroviral spermicide (WHI-07)-loaded gel-microemulsion in the porcine female reproductive tract. J App Toxicol. 2008;28:303-14.

16. das Neves J, Bahia MF. Gels as vaginal drug delivery systems. Int J Pharm. 2006;318:1-14

17. Mashingaidze F, Choonara YE, Kumar P, du Toit LC, Maharaj V, Buchmann E, et al. Poly(ethylene glycol) enclatherated pectin-mucin submicron matrices for intravaginal anti-HIV-1 drug delivery. Int J Pharm. 2016;503:16-28.

18. Shattock RJ, Rosenberg Z. Microbicides: Topical Prevention against HIV. Cold Spring Harb Perspect Med. 2012;2:a007385.

19. Brannon-Peppas L. Design and mathematical analysis of controlled release from microsphere-containing polymeric implants. J Control Release. 1992;20:201-8.

20. Sriamornsak $P$, Wattanakorn N, Takeuchi H. Study on the mucoadhesion mechanism of pectin by atomic force microscopy and mucin-particle method. Carbohydr Polym. 2010;79:54-9.

21. Karnachi AA, Khan MA. Box-behnken design for the optimization of formulation variables of indomethacin coprecipitates with polymer mixtures. Int $\mathrm{J}$ Pharm. 1996;131:9-17.

22. Gupta J, Tao JQ, Garg S, Al-Kassas R. Design and development of an in vitro assay for evaluation of solid vaginal dosage forms. Pharmacol Pharm. 2011;2:289-298.

23. Owen DH, Katz DF. A review of the physical and chemical properties of human semen and the formulation of a semen simulant. J Androl. 2005;26:459-69.

24. Zhang Y, Huo M, Zhou J, Zou A, Li W, Yao C, et al. DDSolver: An add-in program for modeling and comparison of drug dissolution profiles. AAPS J. 2010;12:263-71.

25. Gupta J, Qihai Tao J, Garg S, Al-Kassas R. Design and development of an in vitro assay for evaluation of solid vaginal dosage forms. Pharmacol Pharm. 2011;2:289-98

26. Owen D, Katz D. A Vaginal Fluid Stimulant. Contraception. 1999;59:91-5.

27. Moore JW, Flanner HH. Mathematical comparison of dissolution profiles. Pharm Technol. 1996;20:64-74.

28. Costa P, Sousa Lobo JM. Modeling and comparison of dissolution profiles. Eur J Pharm Sci. 2001;13:123-33.

29. Pillay V, Danckwerts P. Textural profiling and statistical optimization of crosslinked calcium-alginate-pectinate-cellulose acetophthalate gelisphere matrices. J Pharm Sci. 2002;91:2559-70.

30. Kaiser GM, Heuer MM, Frühauf NR, Kühne CA, Broelsch CE. General handling and anaesthesia for experimental surgery in pigs. J Surg Res. 2006;130:73-9.

31. Ndesendo VMK, Pillay V, Choonara YE, du Toit LC, Buchman E, Meyer LCR, et al. Investigation of the physicochemical and Physicomechanical properties of a novel intravaginal bioadhesive polymeric device in the pig model. AAPS PharmSciTech. 2010;11:793-808.

32. Altman GH, Diaz F, Jakuba C, Calabro T, Horan RL, Chen J, et al. Silk-based biomaterials. Biomaterials. 2003;24:401-416.

33. Clark MR, Friend DR. Pharmacokinetics and topical vaginal effects of two tenofovir gels in rabbits. AIDS Res Human Retovir. 2012;28:1458-66.

34. Iriarte G, Gonzalez O, Ferreirós N, Maguregui MI, Alonso RM, Jiménez RM. Validation of a fast liquid chromatography-UV method for the analysis of drugs used in combined cardiovascular therapy. J Chromatogr B. 2009;877:3045-53. 
35. Polson C, Sarkar P, Incledon B, Raguvaran V, Grant R. Optimization of protein precipitation based on effectiveness of protein removal and ionization effect in liquid chromatography-tandem mass spectrometry. J Chromatogr B. 2003;785:263-75.

36. Samanidou VF, Giannakis DE, Papadaki A. Development and validation of an HPLC method for the determination of seven penicillin antibiotics in veterinary drugs and bovine blood plasma. J Sep Sci. 2009;32:1302-11.

37. Ndesendo VMK, Pillay V, Choonara YE, du Toit LC, Meyer LCR, Buchman E, et al. In vivo evaluation of the release of zidovudine and polystyrene sulfornate from a dual intravaginal bioadhesive polymeric device in the pig model. $J$ Pharm Sci. 2011;100:1416-35.

38. Ndesendo VMK, Pillay V, Choonara YE, du Toit LC, Meyer LCR, Buchmann E, et al. In vivo evaluation of the release of zidovudine and polystyrene sulfonate from a dual intravaginal bioadhesive polymeric device in the pig model. 2011;4:1416-1435.

39. Khan GM, Zhu JB. Sustained release coprecipitates and matrix tablet of ibuprofen with polymeric resins: Formulation and in vitro evaluation. J China Pharm Univ. 1998;29:26774.

40. Li S, McCarthy S. Further investigations on the hydrolytic degradation of poly (DLlactide). Biomaterials. 1999;20:35-44.

41. Siepmann F, Eckart K, Maschke A, Kolter K, Siepmann J. Modeling drug release from PVAc/PVP matrix tablets. J Control Release. 2010;141:216-22.

42. Matsuda Y, Minamida Y, Hayashi S-I. Comparative evaluation of tablet lubricants: effect of application method on tablet hardness and ejectability after compression. J Pharm Sci. 1976;65:1155-60.

43. Abu-Izza K, Tambrallo L, Lux R. In vivo evaluation of zidovudine (AZT)-loaded ethylcellulose microspheres after oral administration in beagle dogs. J Pharm Sci. 1997;86:554-9.

44. Koh $\mathrm{Y}$, Haim $\mathrm{H}$, Engelman $\mathrm{A}$. Identification and characterization of persistent intracellular Human Immunodeficiency Virus Type 1 integrase strand transfer inhibitor activity. Antimicrob Agents Chemother. 2011;55:42-49.

45. Miller EA, Beasley DE, Dunn RR, Archie EA. Lactobacilli dominance and vaginal $\mathrm{pH}$ : why is human vaginal microbiome unique. Front Microbiol. 2016. Doi:10.3389/fmicb.2016.01936. 